\title{
Insights into the complexity of the excited states of Eu-doped luminescent materials
}

\author{
Jonas J. Joos ${ }^{1}$, Philippe F. Smet ${ }^{1}$, Luis Seijo ${ }^{2}$, Zoila Barandiarán ${ }^{2}$ \\ ${ }^{1}$ LumiLab, Department of Solid State Sciences, Ghent University, Krijgslaan 281-S1, 9000 Gent, Belgium. \\ Center for Nano- and Biophotonics (NB Photonics), Ghent University, Belgium
${ }^{2}$ Departamento de Química, Instituto Universitario de Ciencia de Materiales Nicolás Cabrera, and Condensed Matter Physics Center (IFIMAC), Universidad Autónoma de Madrid, 28049 Madrid, Spain

This paper was published in Inorg. Chem. Front. (Available online December 17, 2019)

This version is the unedited, accepted version after peer review.

The edited version can be found at:

https://pubs.rsc.org/en/content/articlelanding/2020/qi/c9qi01455a\#!divAbstract

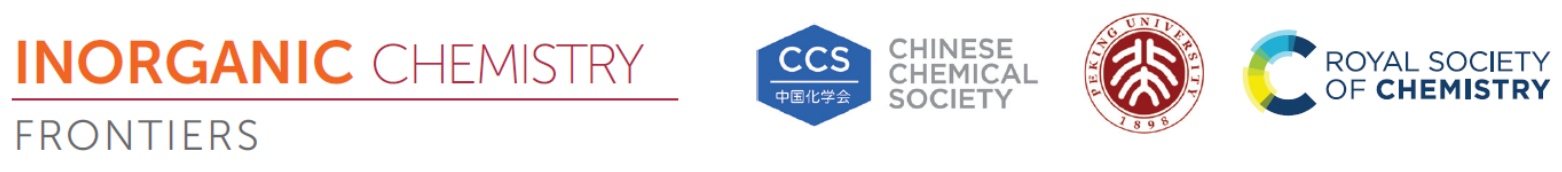

\section{RESEARCH ARTICLE}

Insights into the complexity of the excited states

Cite this: DOI: 10.1039/c9qi01455a of Eu-doped luminescent materials $\uparrow$ 


\title{
Insights on the complexity of the excited states of Eu-doped luminescent materials
}

\author{
Jonas J. Joos ${ }^{1,2}$, Philippe F. Smet ${ }^{1,2}$, Luis Seijo ${ }^{3}$, Zoila BarandiarÁn ${ }^{3}$ \\ ${ }^{1}$ LumiLab, Department of Solid State Sciences, Ghent University, Gent, Belgium \\ ${ }^{2}$ Center for Nano- and Biophotonics (NB Photonics), Ghent University, Gent, Belgium \\ ${ }^{3}$ Departamento de Química, Instituto Universitario de Ciencia de Materiales Nicolás Cabrera, and Condensed Matter \\ Physics Center (IFIMAC), Universidad Autónoma de Madrid, Madrid, Spain.
}

\begin{abstract}
It has always been a spectroscopist's dream to correlate a material's luminescent properties to its microscopic structure, based on reliable structure-property relationships. Electronic structure methods are promising to achieve this goal, yet they are especially challenging in the case of Eu-based materials which are known to feature an exceptionally high density excited states, large spins and severe electron correlation. In this work, state-of-the-art

multiconfigurational $a b$ initio embedded-cluster methods are applied to get a deeper insight into the luminescence mechanisms of $\mathrm{Eu}^{2+}$ and $\mathrm{Eu}^{3+}$-doped phosphors. Regardless of the difficulties, very accurate excitation energies are achieved, reaching $68 \%$ prediction intervals of $300 \mathrm{~cm}^{-1}$, corresponding to an accuracy of $5-10 \mathrm{~nm}$ in the visible wavelength range. Complete configurational coordinate curves are obtained, yielding breathing mode vibrational frequencies and equilibrium bond lengths for all excited states. Moreover, electric dipole transition moments and oscillator strengths are used to calculate absorption spectra. An excellent correspondence with experiment is found. The $a b$ initio calculations give an unprecedented detailed view on the $\mathrm{Eu}^{2+}$ excited state landscape, allowing for an improved understanding of its structure, including the origin of the so-called 'staircase structure' and the role of ligand covalency on the ligand field and exchange splittings. It is found that more covalent host compounds feature higher exchange splittings due to an increased stabilization of high-spin states by the interaction with virtual LMCT states. It

is verified that the equilibrium Eu-ligand bond length contracts upon 4f-5d excitation towards the lowest $5 \mathrm{~d}$ submanifold and that the bond lengths are directly related to the configurational character of the electronic eigenstate. Moreover, comparing ab initio with crystal field calculations proves that a decoupled model for the $\mathrm{Eu}^{2+}$ excited states

is inadequate, making the use of an intermediate coupling scheme compulsory. With this approach, computational design of luminescent materials is getting within reach.
\end{abstract}

\section{Introduction}

Europium is the most studied luminescent activator among the lanthanide elements. It can adopt two possible oxidation states when it is incorporated in inorganic crystals or coordination compounds, $\mathrm{Eu}^{3+}$ and $\mathrm{Eu}^{2+}$, having a $4 f^{6}$ and $4 f^{7}$ ground state configuration respectively. Both oxidation states are of huge relevance for different lighting and display technologies 12 , as well as for storage phosphors or scintillators, e.g. in medical imaging 3 . Especially in fluorescent lamps, $\mathrm{Eu}^{3+}$ has a very rich history thanks to its saturated red emission color, dominated by the ${ }^{5} D_{0} \rightarrow{ }^{7} F_{2}$ emission line that can invariably be found around $611-616 \mathrm{~nm}$ and the presence of low-lying ligand-to-metal chargetransfer (LMCT) states, allowing efficient excitation with the mercury UV emission $(254 \mathrm{~nm})$.

For more modern applications, based on white lightemitting diodes (LEDs), $\mathrm{Eu}^{2+}$ gets into the picture thanks to its low-lying $4 f^{6} 5 d^{1}$ configuration, enabling efficient, while yet spectrally relatively narrow emission across the visible and near-infrared spectral range upon excitation with blue or near-UV light 15 9. The energy difference between the $4 f^{7}$ and $4 f^{6} 5 d^{1}$ configurations is highly dependent on the chemical envi- ronment of the $\mathrm{Eu}^{2+}$ ion. Some coarse systematic is known since a long time, e.g. that this energy difference scales roughly with the covalency of the chemical bond 10 12. The empirical approach is exploited for instance in color point tuning, i.e. provoking subtle color changes upon small chemical substitutions of anions or cations in the host compound $13 / 14$. In contrast to their simplicity and accessibility, these empirical structureproperty relations are however not sufficiently accurate to design new functional materials where the requirements are typically very strict, not only in terms of emission color, but also in terms of thermal stability of the luminescence properties 15 . As an example, the Rec. 2020 standard for a red-emitting phosphor for display applications requires an emission maximum of exactly $630 \mathrm{~nm}$ [16. Deviations from this value should be small, preferably less than $5 \mathrm{~nm}$, otherwise overall energy losses will strongly increase due to color filtering. This deviation corresponds to a tolerance of only $250 \mathrm{~cm}^{-1}$ on computed excited state energies, which is beyond the abilities of empirical models 15 .

The important optical properties of europium derive from its electronic structure, which creates a parti .cularly complex manifold of excited states. These are numerous, highly correlated, and dense sets of states 
of different natures. It is then not surprising that different - often contradictory - analyses of experimental $4 f^{7} \rightarrow 4 f^{6} 5 d$ spectra exist ${ }^{17+27}$ and it is not clear which model correctly grasps the nature of the electronic levels underlying the broad absorption or excitation bands. Furthermore, emission bands are often present in photoluminescence spectra that seem to be related to the presence of $\mathrm{Eu}$, but do not correspond to the expected $4 f^{7} \rightarrow 4 f^{7}$ or $4 f^{6} 5 d^{1} \rightarrow 4 f^{7}$ emissions ${ }^{28,32}$. Because no convincing systematic behavior is found in terms of its occurrence or properties (such as lifetime, thermal behavior, etc.), the origin of these so-called anomalous emissions is typically not explained, or only hypothesized, without valid experimental or theoretical justification. Also, there is a long-lasting question about the mechanism causing the persistent luminescence (afterglow) in many Eu-based phosphors, where it is very difficult to probe the exact charge-carrier dynamics or the presence and role of intrinsic and extrinsic crystal defects by existing experimental techniques 33 35.

The importance and complexity of the excited states of europium in solids justify the present demand for accurate computational techniques that are able to explain and predict the luminescence properties of Eu based phosphors. This requires the ability to perform reliable calculations of not only the energies of excited states of different types, but also of their structural properties, i.e, of their configuration coordinate diagrams in general, and transition probabilities.

We show in this work that embedded cluster multiconfigurational $a b$ initio methods give a detailed account of the complex excited states and spectra of $\mathrm{Eu}^{2+}$ and $\mathrm{Eu}^{3+}$ in the ionic fluorides $\mathrm{CaF}_{2}, \mathrm{SrF}_{2}, \mathrm{BaF}_{2}$, and in the more covalent sulfides $\mathrm{CaS}, \mathrm{SrS}$, and BaS. Quantitative accuracies in the range of $300 \mathrm{~cm}^{-1}$ are achieved, opening the door for designing luminescent materials based on $a b$ initio calculations.

The development and tuning of the used methods belong to the efforts that, apart from empirical rules, have been made to offer computational techniques that can describe excited states of europium. Previous efforts have provided energies at fixed structures rather than configuration coordinate diagrams. Besides, their scope is limited to ligand-field states. On the empirical side, crystal field theory is hard to apply to $\mathrm{Eu}^{2+}$ spectra due to the large density of energy levels involved, complicating the optimizations of parameters from experimental spectra $19 \sqrt[22 / 27 / 36]{39}$. To avoid this, crystal field parameters can be calculated within some theoretical framework, such as the exchange-charge model. This has however not led to convincing results as the $4 f-4 f$ and $4 f-5 d$ Coulomb integrals cannot be calculated in the latter model 40 42. On the first-principles side, several methods have resulted in the calculation of crystal field parameters 434 . For $\mathrm{Eu}^{2+}$, ligandfield density functional theory (LFDFT) has been successful in reproducing $\mathrm{Eu}^{2+}$ spectra for various compounds $45 \mid 46$. In this approach, spin-restricted density functional theory (DFT) Kohn-Sham orbitals are used, with average of configuration (AOC) occupations, to obtain crystal field parameters. These are subsequently used for a conventional crystal field calculation 47 .

This theory, besides being limited to the use of ground state geometries, is focused on studying ligandfield excited states that can be described by the parametrization of the crystal field Hamiltonian, but emissions and more complex phenomena such as charge transfers or interactions of excited states with defects are by definition out of its scope.

Multiconfiguratinal $a b$ initio methods overcome these difficulties and can study configuration coordinate diagrams of ligand-field states ${ }^{48}$ but also states of other nature, like ligand-to-metal charge transfer, 49 inter-valence charge transfer,, 50 dopant-to-host charge transfer, 51 or compensator-to-dopant charge transfer. 52

The multiconfigurational wave function methodology captures very accurately the effect of electron correlation, which is exceptionally strong for f elements. $\underline{.53}$ This, together with the use of state-of-the-art relativistic Hamiltonians and quantum mechanical embedding techniques that provide a reliable description of the interactions between an optically active cluster and the rest of the host, 54 makes the calculations sufficiently accurate to get a good qualitative and quantitative correspondence with experiments.

This methodology has previously shown its value in interpreting and understanding absorption and luminescence spectra of lanthanide ions with simpler (although not simple) excited state manifolds, such as $\mathrm{Ce}^{3+}, \mathrm{Pr}^{3+}, \mathrm{Tb}^{3+}, \mathrm{Tm}^{2+}$ and $\mathrm{Yb}^{2+48 / 54} 58$.

Also, some calculations of this type exist on $4 f^{6} 5 d^{1}$ states of $\mathrm{Eu}^{2+}$ in $\mathrm{CaF}_{2}{ }^{[59}$ and $\mathrm{SrAl}_{2} \mathrm{O}_{4}, \frac{60}{6}$ which focused on the energies of the lowest states at fixed experimental or DFT optimized structures and omitted the study of configuration coordinate diagrams and detailed assignments and analyses of a large number of states.

In this paper, the multiconfigurational $a b$ initio methodology is used to calculate the energies and wave functions, in order to identify and to analyze a very large manifold of excited states of $\mathrm{Eu}^{2+}$ and $\mathrm{Eu}^{3+}$ in the $\mathrm{Ca}, \mathrm{Sr}$, and $\mathrm{Ba}$ series of ionic fluorides and covalent sulfides. This includes i) the calculation of potential energy surfaces, resulting in configuration coordinate diagrams, bond lengths and breathing mode vibrational frequencies and ii) the calculation of electric dipole transition moments and oscillator strengths, resulting in spectral profiles from first principles. This means that the methodology faces here its highest level of complexity so far.

The paper is organized as follows: In Section 2 the method and details of the calculations are summarized. The results on the $4 f^{7}$ and $4 f^{6} 5 d^{1}$ configurations of $\mathrm{Eu}^{2+}$ are shown and discussed in Section 3 . with emphasis on their substructures, ligand field and exchange splittings, excited state bond lengths, and $4 f^{7} \rightarrow 4 f^{6} 5 d^{1}$ spectra; in this section, also the $4 f^{6}$ configuration of $\mathrm{Eu}^{3+}$ is discussed. Finally, conclusions are given in Section 4 . 


\section{Theoretical methods}

The theoretical methods used in this work to study the excited states of europium in fluoride and sulfide crystals are well rooted in the $a b$ initio quantum chemical methodology. Most of them are expressed in terms of complete (infinite) expansions that become feasible by wise, educated, and systematic truncations. Hence they lead to systematic accuracies. We comment next on various truncations accepted in this work; some are quite standard, others stem from the complexity of europium. Details of the calculations are given in the Electronic Supplementary Information.

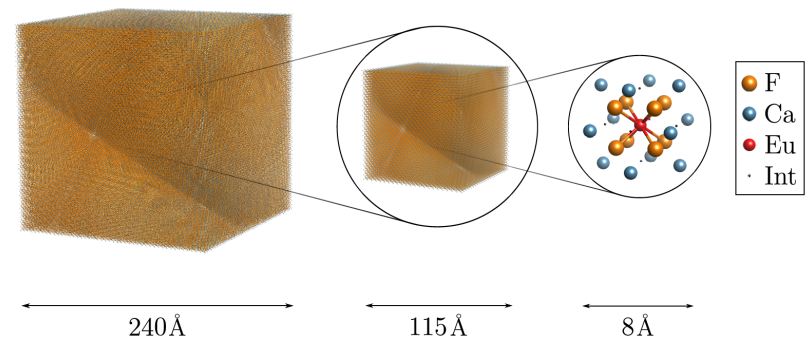

Figure 1 - Graphical representation of the simulated system in the case of the alkaline earth fluorides and how it is divided in the $\left(\mathrm{EuF}_{8} \mathrm{M}_{12}\right)^{2+}(\mathrm{M}=\mathrm{Ca}, \mathrm{Sr}, \mathrm{Ba})$ cluster which is treated at the highest level of theory (right), a large shell represented by an embedding potential which is composed of full-ion AIMPs located at crystal lattice sites (middle) and a thick layer of point charges that terminates the system (left). The indicated distances pertain to $\mathrm{CaF}_{2}$ and are slightly larger for $\mathrm{SrF}_{2}$ and $\mathrm{BaF}_{2}$. A similar partition scheme is used for the sulfides (see text). The interstices in the [100] directions are denoted by Int and indicated by black dots.

At low concentration, europium dopant ions form point defects in the solids we study, creating local (nonperiodic) electronic states that provoke local distortions. Hence the $a b$ initio model potential embeddedcluster approximation (AIMP) ${ }^{61}$ should be a good alternative demanding that boundaries are set for the Eu-containing defect cluster and for the infinite embedding host. From left to right, Fig. 1 zooms in a piece of crystal until the dopant is reached and shows three regions where the methodological efforts are dramatically increased, meaning that quantummechanical rigor, hence expansions, cannot be sacrificed. So, in the distant surrounding region (left) the ions can be treated classically; $[62$ in the intermediate region (middle) quantum-mechanical Pauli antisymmetry related terms that correspond to ascribing singlereference (Hartree-Fock-like) frozen wave functions to the ions, are needed. Finally, in the point-defect cluster (right), state-of-the-art molecular quantum chemical methodology must be used.

Europium is a genuine multivalent open-shell and heavy element. Therefore, correlated methods $63 \mid 64$ and spin-dependent Hamiltonians ${ }^{65 \mid 66}$ must be used to study its electronic structure. In the 90's it was recognized that the multiconfigurational space needed to tackle electron correlation is much larger than that necessary to deal with spin-orbit coupling and, on these grounds, two-step decoupling methods were proposed to address electron correlation in a first step, with a spin-free Hamiltonian, transporting the correlation corrections to the energy spectrum onto a second-step calculation over a smaller multiconfigurational space, through an effective spin-orbit Hamiltonian with reasonable approximations for other spin-dependent interactions. $67 / 68$

But, in spite of the evident benefits of such twostep decoupling, Eu poses a tremendous challenge: the number of excited states is too large, reaching $10^{4}$, and they describe a dense spectrum with basically no energy gaps, which suggests that a dangerous energybased criterion for truncation might be the only possible way towards feasibility. Yet, the two-step strategy serves the purpose in different ways as we comment next.

The total spin $(S)$ remains a good quantum number of the wave functions in the first-step calculations since a spin-free Hamiltonian is used. This enables reasonable spin-based truncations, supported by exploratory calculations, revealing that states with lower than maximal spin occur at increasingly higher energies, this meaning that their spin-orbit coupling effects will be increasingly weaker. Hence, we applied the following spin-based restrictions: only states with $2 S+1 \geq 6$ for $\mathrm{Eu}^{2+}$ and $2 S+1 \geq 5$ for $\mathrm{Eu}^{3+}$ were calculated in the first-step. Therefore, only states with such spin multiplicities were coupled in the second-step spin-orbit calculations. Analyses of the multiconfigurational wave functions corroborate that the ground and many excited states of $\mathrm{Eu}$ can be labeled as $4 f^{7}\left(\mathrm{Eu}^{2+}\right)$ and $4 f^{6}\left(\mathrm{Eu}^{3+}\right)$, where $4 f$ is the main atomic character of the clusters' molecular natural orbitals. The $4 f^{7}$ states do not interact with others describable as single excitations to higher $5 d$ - or $6 s$-like shells, $4 f^{6} 5 d^{1}$ or $4 f^{6} 6 s^{1}$, due to the inversion center of the $\mathrm{Eu} O_{h}$ site symmetry in the fluorite and sulfide hosts $\left(\mathrm{MF}_{2}\right.$ and $\mathrm{MS}, \mathrm{M}=$ $\mathrm{Ca}, \mathrm{Sr}, \mathrm{Ba})$. Thanks to this high symmetry, we could calculate all $O_{h}$-adapted electronic states of the $4 f^{N}$ $(\mathrm{N}=7,6)$ configurations (subject to the spin-based restrictions mentioned above) up to the final spin-orbit second-step. However, the number of $\mathrm{Eu}^{2+}$ gerade states resulting from the $4 f^{6} \times 5 d$ and $4 f^{6} \times 6 s$ couplings is too large, even within the spin restrictions imposed. So, the results of the $4 f^{6}$ manifold of $\mathrm{Eu}^{3+}$ were used to establish a reasonable $4 f^{6}$-sub-shell-based truncation criterion. In particular, the energy gap above the lowest $\mathrm{Eu}^{3+} 4 f^{6}$ term ${ }^{7} F$ (cf. Table S9) suggested restricting the number of roots to those resulting from the following couplings: $4 f^{6}\left({ }^{7} F\right) \times 5 d, 4 f^{6}\left({ }^{7} F\right) \times 6 s$; however, this does not preclude intercalation of states of other configurations such as $4 f^{6}\left({ }^{5} D\right) \times 5 d$ (cf. Section 3.3.2. All we have just mentioned results in limiting the number of roots to be calculated. We focus next on truncations for the multiconfigurational expansions. These are inherent in the used electron correlation methods and determine directly the accuracy and stability of the results. 
The methods we use for electron correlation in the first-step, with a spin-free Hamiltonian, start by calculating a variational, multiconfigurational multistate reference $\frac{6369}{72}$ upon which second order perturbation methods are applied. $\sqrt[64 / 73 \sqrt{76}]{ }$ In the former calculations, the definition of an active orbital set over which a complete CI space is ideally built, is instrumental (CI, Configuration Interaction). Typically, the open-shells and some unoccupied orbitals $\frac{77+79}{79}$ are meaningful and are used, such as those of dominant Eu $4 f, 5 d, 6 s$, and $5 f$ character, in this case. However, the complete active space generated by these meaningful active orbitals is too large and needs to be truncated. So, we performed 7- or 6-electron (for $\mathrm{Eu}^{2+}$ or $\mathrm{Eu}^{3+}$ ) state-average restricted active space self-consistent field calculations (SA-RASSCF) where all occupations of the $4 f$ shell were allowed, whereas only single, double, triple, and quadruple excitations from the $4 f$ to the $5 d, 6 s$, and $5 f$ shells were permitted, compatible with the total spin and site symmetry of the Eu clusters. This truncation should be very close to the complete active space limit. Analyses of the SA-RASSCF wave functions and energy curves of the gerade excited states of $\mathrm{Eu}^{2+}$, revealed the occurrence of expected impurity-states ascribable to intraconfigurational excitations of Eu: $4 f^{6} 5 d e_{g}^{1}, 4 f^{6} 5 d t_{2 g}^{1}$, and $4 f^{6} 6 s^{1}$ configurations, but they also showed the presence of more delocalized states, so-called impurity-trapped-excitons (ITE) because the excited electron density spreads close but beyond first neighbors: 80$] f^{6} \phi_{\mathrm{ITE}}^{1}$. These ITE states (of $a_{1 g}$ symmetry) were found in the studied energy range for the fluoride hosts, while $4 f^{6} 6 s^{1}$ states were obtained in the sulfides. States of different configurations show significant differences in bond lengths, as discussed in Section 3.3.5. Here again, the very high density of states of Eu poses further difficulties that (only) affect some spin-sextet $\mathrm{Eu}^{2+}$ excited states that lie high in energy, in the middle of a continuum of other states. So, interactions between some impurity and ITE spin-sextet states resulted in deformations of their potential energy surfaces, including, in some cases, sharp avoided crossings. As mentioned above, the SA-RASSCF states obtained are allowed to interact in a larger space in the so-called multistate second order perturbational calculations (MS-RASPT2), which brings necessary dynamic correlation corrections after time-consuming and resources-demanding MS-RASPT2 calculations Even though this multistate-reference method is prepared to deal with avoided crossings, and it generally does, we found irregular shapes in the potential energy surfaces in the form of small spikes and/or bumps close to the sharp avoided crossings of their parent SA-RASSCF states. The irregularities are smoothed slightly when the number of states allowed to interact was increased, but they did not disappear. Therefore, we decided to simply omit those spin-sextet states showing irregular potential energy surfaces or lying above them in the final second-step spin-orbit calculations. High energy sextets were also omitted from the spin-orbit calculation for the BaS host due to difficulties in their assignment to irreducible representations of the octahedral

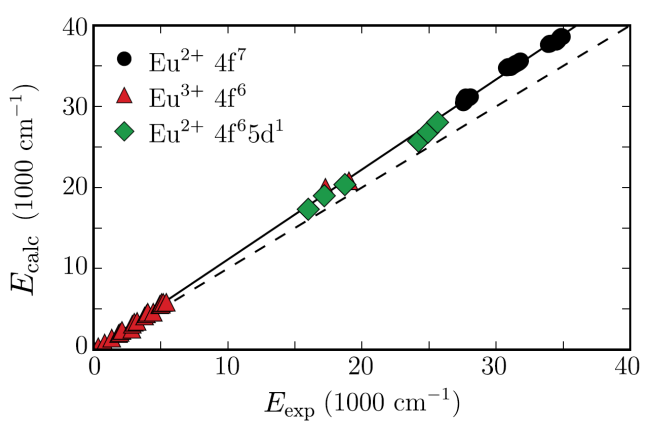

Figure 2 - Comparison of calculated (this work) and experimental ${ }^{[29 \mid 84]}$ excited state energies of $\mathrm{Eu}^{2+}$ and $\mathrm{Eu}^{3+}$ in fluorides and sulfides. The dashed line represents perfect match between theory and experiment; the solid black line corresponds to $E_{\exp }=$ $0.9 E_{\text {calc }}$. See Table S15 for detailed data.

point group.

Detailed results can be found in the Electronic Supplementary Information: spin-orbit-free results (SARASSCF and MS-RASPT2) are presented in Tables S1 to S6 and Figures S1 and S2, those of the final spinorbit calculations are given in Tables S9 to S14, Figure S3 and below. The calculations of this work have been done using the MOLCAS programs. ${ }^{81}$ Relativistic basis sets from Refs. 82 and 83 have been used to expand the molecular orbitals from the cluster.

From the comments in this section, pertaining to all the different truncations assumed in this work, it can be expected that the final spin-orbit results are robust and reveal systematic errors, increasing with energy, when confronted with direct experimental results. Accordingly, we compared theoretical and available experimental excited state energies (Fig. 2 and Table S15) and found a systematic overestimation, which is in consonance with the systematic methodological truncations discussed above. The deviation increases linearly with energy and suggests a $90 \%$ scaling of the theoretical values: $E_{\text {exp }} \simeq E_{\text {calc }}^{\text {sc }}=0.9 E_{\text {calc. }}$. In the following, the results will be discussed based on these scaled energies; the corresponding raw calculated data are available in the Electronic Supplementary Information.

Statistical analysis of the data shows a $68 \%$ prediction interval of only $300 \mathrm{~cm}^{-1}(40 \mathrm{meV})$. This is a figure of merit for which the computational design of luminescent materials for high-end applications, as described above, comes in reach. The achieved precision outperforms prevailing empirical rules based on interand extrapolation of known experimental data, e.g. to predict the $4 f-5 d$ transition energy of a lanthanide ion when this quantity is known for another ion in the same host. Typical $68 \%$ prediction intervals for empirical rules amount to $800-4000 \mathrm{~cm}^{-1}(100-500 \mathrm{meV})^{[15}$. 
Table 1 - Spectroscopic constants of $\mathrm{Eu}^{2+}$-doped alkaline earth difluorides and sulfides. Experimental values for the lowest $4 f-5 d$ transition have been accurately determined from zero-phonon lines in $\mathrm{CaF}_{2}$ (Ref. 88), $\mathrm{SrF}_{2}$ (Ref. 85), CaS (Ref. 86), and SrS (Ref. 87); they are estimated from band shapes in $\mathrm{BaF}_{2}$ (Ref. 85) and BaS (Ref. 29), and are shown in italics. The ligand field splitting parameter $\epsilon_{\mathrm{lfs}}^{\mathrm{HS}}$ is defined in Eq. 1 Exchange splitting parameters $\epsilon_{\text {exch }}^{e g}$ are given for fluorides and $\epsilon_{\text {exch }}^{t_{2 g}}$ for sulfides (Eq. 2]. Energies are given in $\mathrm{cm}^{-1}$ and distances in $\AA$.

\begin{tabular}{|c|c|c|c|c|c|c|}
\hline & $\mathrm{CaF}_{2}$ & $\mathrm{SrF}_{2}$ & $\mathrm{BaF}_{2}$ & $\mathrm{CaS}$ & $\mathrm{SrS}$ & $\mathrm{BaS}$ \\
\hline \multicolumn{7}{|c|}{ Eu-F/Eu-S equilibrium distances } \\
\hline $4 f^{7}\left({ }^{8} S\right)$ & 2.388 & 2.470 & 2.558 & 2.876 & 2.975 & 3.087 \\
\hline $4 f^{6} 5 d^{1}\left(1 \Gamma_{8 g}\right)$ & 2.373 & 2.451 & 2.535 & 2.849 & 2.945 & 3.037 \\
\hline \multicolumn{7}{|c|}{$\mathrm{EuF}_{8} / \mathrm{EuS}_{6}$ breathing mode vibrational frequencies } \\
\hline $4 f^{7}\left({ }^{8} S\right)$ & 423 & 370 & 321 & 286 & 273 & 203 \\
\hline $4 f^{6} 5 d^{1}\left(1 \Gamma_{8 g}\right)$ & 428 & 370 & 328 & 283 & 263 & 196 \\
\hline \multicolumn{7}{|l|}{ Energy differences } \\
\hline$E_{\text {calc }}^{\text {sc, } f d}$ & 23150 & 24145 & 25240 & 15580 & 17080 & 18320 \\
\hline$E_{\exp }^{f d d}$ & 24215 & 24925 & 25636 & 15995 & 17191 & 18729 \\
\hline$E_{\text {calc }}^{\operatorname{sc}, f d}-E_{\text {exp }}^{f d}$ & -1065 & -780 & -396 & -415 & -110 & -410 \\
\hline$\epsilon_{\mathrm{lfs}}^{\mathrm{HS}}$ & 18790 & 16220 & 14460 & 22370 & 20170 & 16070 \\
\hline & 16480 & 14150 & 12670 & & & \\
\hline$\epsilon_{\mathrm{lfs}}^{\mathrm{HS}}-\epsilon_{\mathrm{lfs}, \exp }^{\mathrm{HS}}$ & 2310 & 2070 & 1790 & & & \\
\hline$\epsilon_{\mathrm{exch}}^{e_{g}}$ or $\epsilon_{\mathrm{exch}}^{t_{2 g}}$ & 4360 & 4410 & 5210 & 7090 & 7090 & 7820 \\
\hline
\end{tabular}

\section{Results and discussion}

\section{1 $\mathrm{Eu}^{2+}$ excited states}

The computed configuration coordinate diagrams of the $\mathrm{Eu}^{2+}$ active center in the six host crystals $\mathrm{CaF}_{2}$, $\mathrm{SrF}_{2}, \mathrm{BaF}_{2}, \mathrm{CaS}, \mathrm{SrS}$, and BaS, are shown in Fig. 3 . These are potential energy curves along the breathing mode that include spin-orbit coupling effects (detailed analyses of the spin-free results can be found in the Electronic Supplementary Information). A summary of results is presented in Table 1. We will use them in the next sections, where we discuss the $4 f^{7}$ and $4 f^{6} 5 d^{1}$ manifolds of $\mathrm{Eu}^{2+}$ and the $4 f^{6}$ manifold of $\mathrm{Eu}^{3+}$, as well as the systematic variations originating from cation substitutions (from Ca to $\mathrm{Sr}$ to $\mathrm{Ba}$ ) and by altering the covalency of the chemical bonds and the ligand coordination (from $\mathrm{F}$ to $\mathrm{S}$ ).

\subsection{The $\mathbf{E u}^{2+} 4 f^{7}$ configuration}

The $4 f^{7}$ configuration yields the ground state of the $\mathrm{Eu}^{2+}$ ion, more specifically the ${ }^{8} S$ term, which is composed of only one configuration where all seven $f$ electrons have their spin parallel. In $O_{h}$ symmetry, spinorbit coupling splits this level into a quartet, $\Gamma_{8 u}$, and two Kramers doublets, $\Gamma_{6 u}$ and $\Gamma_{7 u}$; however, the splitting of at most $0.2 \mathrm{~cm}^{-1}$ has no measurable influence on optical and luminescence properties.

Eu-ligand equilibrium distances (cf. Table 1) increase for crystals with larger alkaline earth ions. Sulfides feature larger bond lengths than the fluorides, irrespective of their smaller coordination number (6 vs. 8). In all cases, the ground state bond length is slightly smaller than the bond lengths for the higher-lying spinsextets: about $0.002 \AA$ and $0.006 \AA$ for the fluorides and sulfides, respectively. Few experimental data exist
Table 2 - Average energies for the lowest terms of the $4 \mathrm{f}^{7}$ manifold of $\mathrm{Eu}^{2+}$ in $\mathrm{CaF}_{2}$ and $\mathrm{SrF}_{2}$. Experimental values obtained by two-photon absorption spectroscopy from Ref. 84. All values in $\mathrm{cm}^{-1}$.

\begin{tabular}{|c|c|c|c|c|c|c|c|c|c|}
\hline & \multicolumn{3}{|c|}{$\mathrm{CaF}_{2}: \mathrm{Eu}^{2+}$} & \multicolumn{3}{|c|}{$\mathrm{SrF}_{2}: \mathrm{Eu}^{2+}$} & \multicolumn{3}{|c|}{$\mathrm{BaF}_{2}: \mathrm{Eu}^{2+}$} \\
\hline & $E_{\text {calc }}^{\mathrm{sc}}$ & $E_{\exp }$ & diff. & $E_{\text {calc }}^{\mathrm{sc}}$ & $E_{\exp }$ & diff. & $E_{\text {calc }}^{\mathrm{sc}}$ & $E_{\exp }$ & diff. \\
\hline${ }^{8} S$ & 0 & 0 & 0 & 0 & 0 & 0 & 0 & & \\
\hline${ }^{6} \mathrm{P}$ & 27726 & 27804 & -78 & 27849 & 27901 & -52 & 28057 & & \\
\hline $2 \Gamma_{8 u}$ & 27398 & 27558 & -160 & 27519 & 27654 & -135 & 27728 & 27727 & 1 \\
\hline $2 \Gamma_{7 u}$ & 27408 & 27564 & -156 & 27527 & 27658 & -132 & 27734 & 27729 & 5 \\
\hline $3 \Gamma_{8 u}$ & 27743 & 27588 & 155 & 27866 & 27672 & 194 & 28075 & 27738 & 337 \\
\hline $2 \Gamma_{6 u}$ & 27948 & 27959 & -11 & 28075 & 28066 & 9 & 28285 & & \\
\hline $4 \Gamma_{8 u}$ & 27959 & 27999 & -41 & 28085 & 28098 & -14 & 28292 & & \\
\hline $3 \Gamma_{7 u}$ & 27978 & & & 28100 & & & 28304 & & \\
\hline${ }^{6} I$ & 31631 & 31382 & 249 & 31767 & 31491 & 276 & 31981 & & \\
\hline${ }^{6} D$ & 34290 & 34474 & -184 & 34435 & 34496 & -61 & 34667 & & \\
\hline${ }^{6} G+{ }^{6} F$ & 45249 & & & 45437 & & & 45693 & & \\
\hline${ }^{6} \mathrm{H}$ & 50602 & & & 50790 & & & 51043 & & \\
\hline
\end{tabular}

on the exact geometries and bond lengths of dopants or defects, yet the Eu-F distance for $\mathrm{CaF}_{2}: \mathrm{Eu}^{2+}$ was found to be $2.413 \AA \pm 0.027 \AA$ from extended X-ray absorption fine structure (EXAFS) ${ }^{90}$, which agrees with the calculated value of $2.388 \AA$.

Overlapping with the dense $4 f^{6} 5 d^{1}$ manifold (see Sec. 3.3), excited terms of the $4 f^{7}$ configuration can be found at energies above $25000 \mathrm{~cm}^{-1}$ (Fig. 3, in black), of which the ${ }^{6} P,{ }^{6} I$ and ${ }^{6} D$ are the first in energy (Tab 2). Unless in some exceptional cases, such as sulfates or ternary fluorides ${ }^{91}$, this part of the $4 f^{7}$ manifold cannot be straightforwardly studied from luminescence spectroscopy due to the overlapping $4 f^{6} 5 d^{1}$ manifold ${ }^{92}$. An exception is when so-called Fano antiresonances are present in the excitation spectrum, however this is rarely observed 21. A more practical approach to study higher excited states of the $4 f^{7}$ configuration is by two-photon absorption spectroscopy, a technique where $4 f-4 f$ transitions are allowed ${ }^{93 \mid 94} . \mathrm{CaF}_{2}: \mathrm{Eu}^{2+}$ and $\mathrm{SrF}_{2}: \mathrm{Eu}^{2+}$ happen to be the earliest test cases for this technique, proving the existence of higher-lying $4 f^{7}$ levels in accordance to the iso-electronic $\mathrm{Gd}^{3+}$ ion ${ }^{95 \mid 96}$. Later, Downer et al., carried out a meticulous study on the same crystals, resulting in the accurate determination of the levels of the $4 f^{7}$ manifold of $\mathrm{Eu}^{2+}$ in these compounds up to $35000 \mathrm{~cm}^{-184}$. The average term energies that were experimentally found are compared with our ab initio results in Table2. The ${ }^{6} G$ and ${ }^{6} F$ terms around $45000 \mathrm{~cm}^{-1}$ are almost degenerate, making it impossible to separate them. Table 2 also compares the energies of the individual lines that originate from the ${ }^{6} P$ term with the computed levels. From this analysis, it is clear that the multiplet splittings due to the interaction with the chemical environment and spin-orbit coupling can be reproduced by the calculation, showing deviations in the order of $100 \mathrm{~cm}^{-1}$ with a maximum of $337 \mathrm{~cm}^{-1}$.

\subsection{The $\mathbf{E u}^{2+} 4 f^{6} 5 d^{1}$ configuration}

$4 f^{N-1} 5 d^{1}$ configurations of lanthanide ions have in general a very complicated structure due to the large number of states (e.g. 30030 in $\mathrm{Eu}^{2+}$ ). They are more difficult to study than $4 f^{N}$ configurations be- 

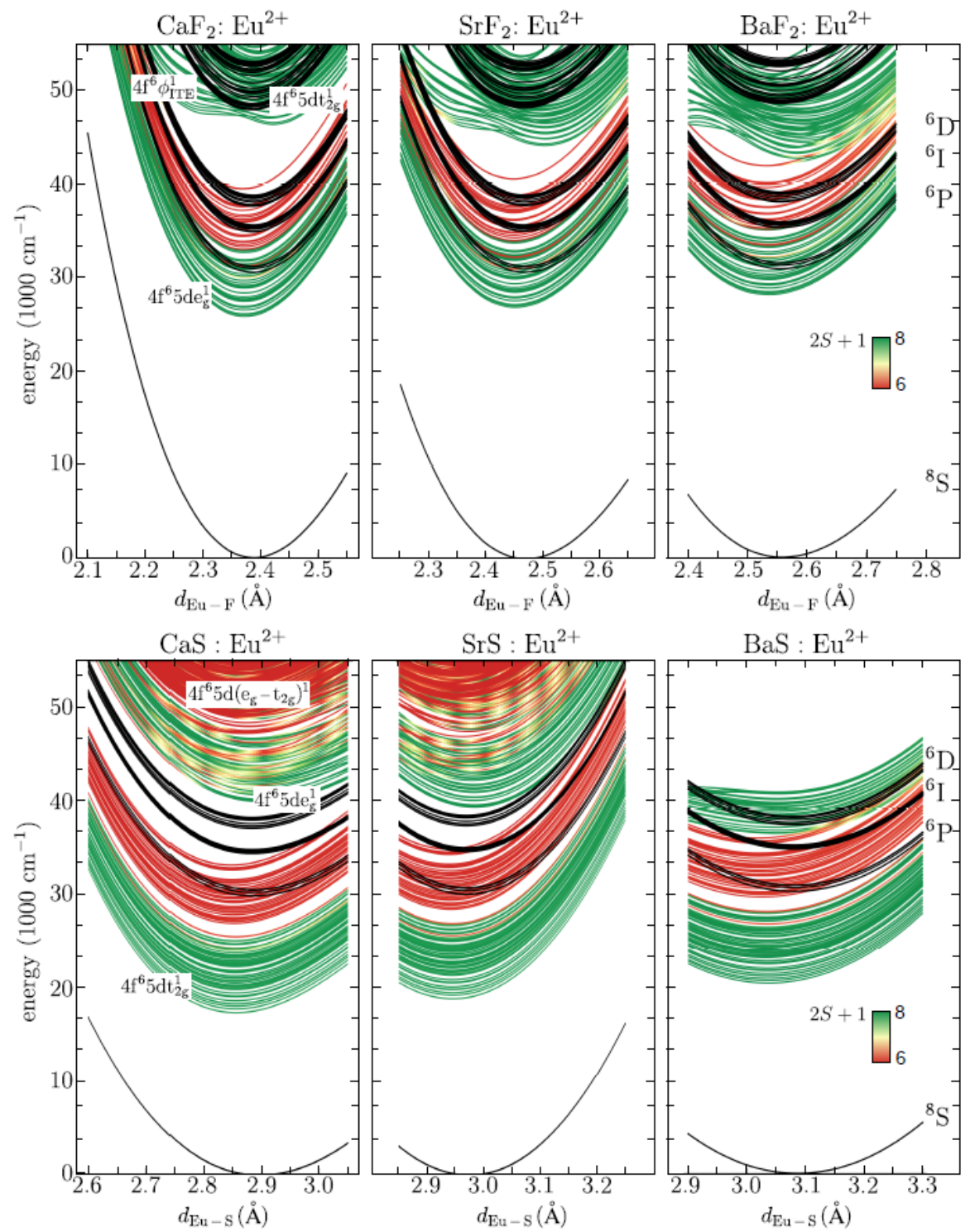

Figure 3 - Potential energy curves for the $\mathrm{Eu}^{2+}$ impurity in the alkaline earth fluorides $\mathrm{CaF}_{2}, \mathrm{SrF}_{2}$ and $\mathrm{BaF}_{2}$ and sulfides CaS, SrS and BaS. The black curves show the levels that correspond to the $4 f^{7}$ configuration, while colored curves originate from the excited $4 f^{6}(5 d, 6 s)^{1}$ configurations. Their color represents the spin-character of the eigenstates, ranging from green for pure spin-octets to red for pure spin-sextets.

cause $4 f-5 d$ spectra feature relatively broad bands, whereas $4 f-4 f$ spectra show atomic-like sharp lines. Parametrized (semi-)empirical approaches are hence impossible or at best impractical to study the details of these configurations 97 . Yet, especially in the case of the $\mathrm{Eu}^{2+}$ ion, a good understanding of the $4 f^{6} 5 d^{1}$ configuration is essential because many modern technologies rely on it.

It is empirically known that the onset of the $\mathrm{Eu}^{2+}$ $4 f^{6} 5 d^{1}$ manifold with respect to the $4 f^{7}$ ground state, and hence the emission color of the $\mathrm{Eu}^{2+}$ luminescence, depends strongly on the chemical nature of the ligands. Ligands that form ionic bonds, such as fluorides, typically give rise to a relatively high-lying $4 f^{6} 5 d^{1}$ manifold corresponding to violet or near-UV emission. Ligands that form more covalent bonds redshift the $4 f^{6} 5 d^{1}$ manifold, allowing a color tuning across the visible spectral range. E.g. the binary sulfides CaS:Eu ${ }^{2+}$ and
$\mathrm{SrS}: \mathrm{Eu}^{2+}$ give rise to a red and orange emission respectively, ${ }^{87}$ which in turn illustrates that cations also play a role. However, knowledge on systematic behavior of $\mathrm{Eu}^{2+}$ luminescence is only qualitative, which limits its practical use. This is specially so when industry requirements are very strict $12|98| 99$.

In this section we aim at providing a quantitative understanding of the $4 f^{6} 5 d^{1}$ configuration of $\mathrm{Eu}^{2+}$. Fig. 3 shows the calculated potential energy surfaces for the $\mathrm{Eu}^{2+} 4 f^{6} 5 d^{1}$ states of the studied materials up to around $50000 \mathrm{~cm}^{-1}$. The host effect on the $4 f^{6} 5 d^{1}$ manifold onset is represented in Table 1 with the zerophonon line energies of the lowest $4 f^{6} 5 d^{1}$ state: the calculated $E_{\text {calc }}^{\mathrm{sc}, f d}$ and the experimental $E_{\text {exp }}^{f d}$. It is clear that the calculated host effect is qualitatively as expected, i.e. a red shift from fluorides to sulfides, which is attributed to the increase in the covalence of the chemical bond 100 . But it is also quantitatively correct, 
with differences between the computed and experimental zero-phonon lines of the order of $1000 \mathrm{~cm}^{-1}$ or less, which evidences the quality of the computed wave functions and their ability to reproduce the host effects.

\subsubsection{Impurity trapped excitons}

Before the submanifolds of $4 f^{6} 5 d t_{2 g}^{1}, 4 f^{6} 5 d e_{g}^{1}$ and highor low-spin characters are discussed, the double-well structure found in the higher energy ranges of the fluoride hosts is examined. Similar electronic structures have been found before in other lanthanide and actinide doped materials ${ }^{80 \mid 101}$ and they result from the interaction between levels with dominant $4 f^{6} 5 d^{1}$ configurational character and impurity-trapped-exciton levels (ITE). The latter have a dominant $4 f^{6} \phi_{\text {ITE }}^{1}$ configurational character, where the molecular orbital $\phi_{\text {ITE }}$ describes an electron delocalized towards the nearby interstitial sites of the fluorite structure, almost ionized but trapped outside the $\mathrm{EuF}_{6}$ moiety. This results in ITE potential energy curves having very small Eu-F equilibrium distances as compared with regular $\mathrm{Eu}^{2+}$ states, only slightly larger than in $\mathrm{Eu}^{3+} 4 f^{6}$ states. The interaction between $4 f^{6} 5 d^{1}$ and ITE states of equal symmetry and the avoided crossing according to the Wigner-Von Neumann rule explains the double well.

\subsubsection{Structure of the $4 f^{6} 5 d^{1}$ manifold}

The structure of the $\mathrm{Eu}^{2+} 4 f^{6} 5 d^{1}$ manifold in the six doped materials under study results from four crowded submanifolds. The lowest two show clear configurational and spin character whereas the highest two overlap and interact significantly. We will discuss this for CaS for the sake of simplicity; similar descriptions hold in the other hosts, with obvious $5 d t_{2 g} / 5 d e_{g}$ changes in the fluorides.

In $\mathrm{CaS}: \mathrm{Eu}^{2+}$, the lowest two submanifolds have a common configurational character $4 f^{6}\left({ }^{7} F\right) 5 d t_{2 g}^{1}$ and, associated to it, common local structure parameters (bond lengths and vibrational frequencies, see Section 3.3.5. These submanifolds are made of all states resulting from the coupling between the well shielded $4 f^{6}$ inner electronic shell in all its states derived from the ${ }^{7} F$ atomic term (strongly split by spin-orbit coupling and weakly split by the crystal field) and the outer $5 d t_{2 g}$ electron. The first submanifold includes the states with high-spin coupling (HS, $2 S+1=8$ ) and the second the higher energy states with low-spin coupling (LS, $2 S+1=6$ ), shown respectively in green and red in Fig. 3. The spin-orbit coupling between the two submanifolds turns out to be very small.

The coupling between $4 f^{6}\left({ }^{7} F\right)$ and the ligand-field excited $5 d e_{g}$ electron leads, initially, to two similar sets of separated HS and LS states that start at around $40000 \mathrm{~cm}^{-1}$ above the ground state. But the internal excitation within the $4 f^{6}$ shell into $4 f^{6}\left({ }^{5} D\right)$ is similar in energy to the $5 d t_{2 g^{-}}-5 d e_{g}$ ligand-field splitting and, as a result, LS states of $4 f^{6}\left({ }^{5} D\right) 5 d t_{2 g}^{1}$ character lie in the same energy window than HS states of $4 f^{6}\left({ }^{7} F\right) 5 d e_{g}^{1}$ character, and their mixing is strong. All of this results in a third submanifold of states with strong HSLS mixing and strong $4 f^{6}\left({ }^{5} D\right) 5 d t_{2 g}^{1}-4 f^{6}\left({ }^{7} F\right) 5 d e_{g}^{1}$ configurational mixing and, associated to the latter, more complex bond lengths and vibrational frequencies (see Section 3.3.5). The fourth submanifold has a more clear LS character.

This discussion also holds for SrS. For BaS and the fluorides, it holds as well, but the absence of high energy spin-sextets in the spin-orbit calculations (Sec. 2) prevents the spin and configurational mixing of the third submanifold, hence the dominant green color in their high energy curves. However, as we will see below, the impact of these sextets in the absorption spectrum is negligible.

According to the crowded submanifolds resulting from combining main spin and configurational characters, namely $\mathrm{HS}$ and LS, $4 f^{6}\left({ }^{7} F\right) 5 d e_{g}^{1}$ and $4 f^{6}\left({ }^{7} F\right) 5 d t_{2 g}^{1}$, it is possible and convenient to introduce a few experimentally meaningful parameters. We will consider HS or LS states those whose spin-orbit wave function shows $\geq 90 \%$ spin octet or sextet character, respectively.

\subsubsection{Ligand field splitting}

Let us first define a high-spin ligand field splitting parameter $\epsilon_{\mathrm{lfs}}^{\mathrm{HS}}$, as the positive energy difference between the averages of the equilibrium energies of the HS $4 f^{6}\left({ }^{7} F\right) 5 d e_{g}^{1}$ and $4 f^{6}\left({ }^{7} F\right) 5 d t_{2 g}^{1}$ states:

$$
\begin{aligned}
\epsilon_{\mathrm{lfs}}^{\mathrm{HS}} & =\mid \frac{1}{N_{e_{g}}^{\mathrm{HS}}} \sum_{i=1}^{N_{e_{g}}^{\mathrm{HS}}} E\left[4 f^{6}\left({ }^{7} F\right) 5 d^{1} e_{g}, \mathrm{HS}\right]_{i} \\
& -\frac{1}{N_{t_{2 g}}^{\mathrm{HS}}} \sum_{i=1}^{N_{t_{2 g}}^{\mathrm{HS}}} E\left[4 f^{6}\left({ }^{7} F\right) 5 d^{1} t_{2 g}, \mathrm{HS}\right]_{i} \mid .
\end{aligned}
$$

This parameter informs on the ligand field strength on the $5 d$ shell, but it should not be identified with traditional crystal field theory parameters like $10 D q$, which is the splitting of one-electron levels, nor is it a configurational average parameter. Furthermore, we make averages only on HS levels because they have nonzero transition probability from the $4 \mathrm{f}^{7}$ ground state (see below). The computed values are given in Table 1 .

Experimentally, the ligand field splitting as defined by Eq. 1 can be approximated by the difference of the average values of both excitation bands. This is denoted as $\epsilon_{\mathrm{lfs}, \exp }^{\mathrm{HS}}$ in Table 1. This approach is very approximate because spectral shapes change as a function of temperature or doping concentration, therefore the empirical values are written in italic. Furthermore, this approach is not applicable to the sulfides because of low-lying excitations of the host compound (see below). Comparing the experimental values with the computed ligand field splittings, it is clear that a constant offset of approximately $2000 \mathrm{~cm}^{-1}$ is found between experimental and computed parameters. Regardless of this offset, which is believed to be the cumulative effect 
of systematic errors in the calculated energies and the difficulty to empirically obtain this parameter, the systematic trend is correctly reproduced.

From the $\epsilon_{\mathrm{lfs}}^{\mathrm{HS}}$ values in Table 1, a drop can be observed for increasing size of the alkaline earth cation, hence with the Eu-ligand bond length, both in the difluorides and the sulfides. This corresponds to the wellknown qualitative trend 102 . Quantitatively, $\epsilon_{\mathrm{lfs}}^{\mathrm{HS}}$ drops from $\mathrm{CaF}_{2}$ to $\mathrm{SrF}_{2}(14 \%)$ to $\mathrm{BaF}_{2}(11 \%)$ and from CaS to $\mathrm{SrS}(10 \%)$ to $\mathrm{BaS}(20 \%)$. It is lower in the difluorides than in the sulfides, in spite of the shorter Eu-ligand distances (16\% lower in $\mathrm{CaF}_{2}$ than in $\mathrm{CaS}, 20 \%$ lower in $\mathrm{SrF}_{2}$ than in $\mathrm{SrS}$, and $10 \%$ lower in $\mathrm{BaF}_{2}$ than in $\mathrm{BaS}$ ) which results from the balance of several factors: Eu-ligand distance, coordination number, ligand oxidation state, and bond covalency.

\subsubsection{Exchange splitting}

It is also interesting to define exchange splitting parameters $\epsilon_{\text {exch }}^{e_{g}}$ (for the fluorides) and $\epsilon_{\text {exch }}^{t_{2 g}}$ (for the sulfides), as the differences between the averages of the equilibrium energies of the LS and HS $4 f^{6}\left({ }^{7} F\right) 5 d e_{g}$ states, and the LS and HS $4 f^{6}\left({ }^{7} F\right) 5 d t_{2 g}$ states, respectively:

$$
\begin{aligned}
\epsilon_{\mathrm{exch}}^{\gamma} & =\frac{1}{N_{\gamma}^{\mathrm{LS}}} \sum_{i=1}^{N_{\gamma}^{\mathrm{LS}}} E\left[4 f^{6}\left({ }^{7} F\right) 5 d^{1} \gamma, \mathrm{LS}\right]_{i} \\
& -\frac{1}{N_{\gamma}^{\mathrm{HS}}} \sum_{i=1}^{N_{\gamma}^{\mathrm{HS}}} E\left[4 f^{6}\left({ }^{7} F\right) 5 d^{1} \gamma, \mathrm{HS}\right]_{i} .
\end{aligned}
$$

with $\gamma=\mathrm{e}_{g}, \mathrm{t}_{2 g}$. These parameters inform of the strength of the $4 f-5 d e_{g}$ and $4 f-5 d t_{2 g}$ interactions. Only states with high spin-purity $(\geq 90 \%)$ were used in Eq2. The definition of $\epsilon_{\text {exch }}^{\gamma}$ relies on $S$ being a good (at least by approximation) quantum number. If this condition is not fulfilled, e.g. for the higher submanifolds of the $4 f^{6} 5 d^{1}$ configuration of $\mathrm{Eu}^{2+}$, or in the $4 f^{N-1} 5 d^{1}$ configurations of heavier lanthanides such as $\mathrm{Tm}^{2+58}$ or $\mathrm{Yb}^{2+48}$, this parameter loses its meaning. The numerical values for $\mathrm{Eu}^{2+}$ are shown in Table 1 .

The $4 f-5 d$ exchange splittings only slightly increase upon enlarging the unit cell, but they experience a significant increase from the difluorides (around $4500 \mathrm{~cm}^{-1}$ ) to the sulfides (around $7500 \mathrm{~cm}^{-1}$ ). This is opposite to the experimentally shown decrease of the $4 f-5 d$ exchange splitting with the covalency of the host in the $4 f^{7} 5 d^{1}$ configuration of $\mathrm{Tb}^{3+} .103 \mid 104$

The opposite effect of increasing covalency on the exchange splitting of $\mathrm{Eu}^{2+}$ and $\mathrm{Tb}^{3+}$, hence of $4 f^{6} 5 d^{1}$ and $4 f^{7} 5 d^{1}$ configurations, can be understood by analyzing the role of virtual ligand-to-metal charge transfer (LMCT) excitations or valence bond (VB) configurations, which are more important in more covalent bonds. This mechanism is displayed in Fig. 4. In effect, in $\mathrm{Eu}^{2+}$-doped sulfides for instance, $\mathrm{S} 3 p \rightarrow \mathrm{Eu} 4 f$ virtual excitations create LMCT configurations of the type $\mathrm{Eu}^{+}\left(4 f^{7} 5 d^{1}\right) \times \mathrm{S}^{-}\left(3 p^{5}\right)$. In reality, the $\mathrm{S}-3 p$ hole will distribute among the ligands according to a molecular orbital, hence the notation $4 f^{7} 5 d^{1} \underline{L}$ is used to denote the virtual LMCT configuration, where $\underline{L}$ denotes

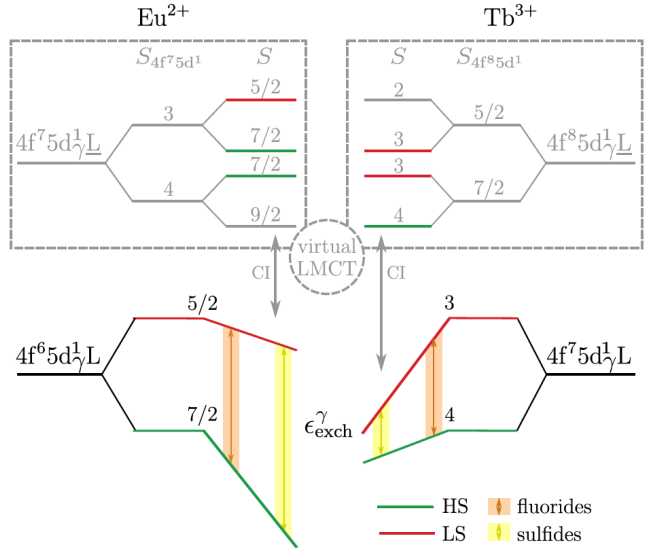

Figure 4 - Schematic representation of how configuration interaction (CI) with virtual ligand-to-metal charge transfer (LMCT) states increases or decreases the exchange splitting of the $4 f^{N-1} 5 d^{1}$ configurations of $\mathrm{Eu}^{2+}(N=7)$ and $\mathrm{Tb}^{3+}(N=8)$ respectively. $L / \underline{L}$ denote a ligand shell which is full/contains one hole.

the hole in a ligand molecular orbital. A state of the $4 f^{N-1} 5 d^{1}$ configuration with total spin $S$ will experience a stabilization from the virtual LMCT states with the same total spin. States with different spins are hence affected differently by the virtual LMCT configuration. This results in the exchange splitting being effectively influenced by the location (related to the covalency of the ligand) as well as the nature of the LMCT states (which $S$ values occur, related to $N$ ). In the following, this effect is elaborated for $\mathrm{Eu}^{2+}$ and $\mathrm{Tb}^{3+}$.

In case of $\mathrm{Eu}^{2+}$, the values of the total spin $S$ of the virtual LMCT states results from the coupling $S_{4 f^{7} 5 d^{1}} \times S_{\underline{L}}$, which can take the values 4,3 (lower spins are not considered because their higher energy and smaller interaction strength) and $1 / 2$, respectively. The coupling $4 \times \frac{1}{2}$ produces $S=\frac{9}{2}, \frac{7}{2}$, and the coupling $3 \times \frac{1}{2}$ produces $S=\frac{7}{2}, \frac{5}{2}$. The splitting of the $4 f^{7} 5 d^{1} \underline{L}$ configuration according to $S_{4 f^{7} 5 d^{1}}$ and, subsequently, $S$ is shown in Fig. 4. The LMCT configuration results in a larger number of $S=\frac{7}{2}$ virtual excitations than $S=\frac{5}{2}$ ones, providing an extra stabilization to the HS octet states $(S=7 / 2)$ with respect to the LS sextet states $(S=5 / 2)$. The virtual LMCT configuration occurs at lower energy for more covalent ligands, consequently its effect on the LS-HS energy difference is larger in sulfides than in fluorides. $\mathrm{Eu}^{2+}$ shows hence a larger exchange splitting in sulfides then in fluorides.

In case of $\mathrm{Tb}^{3+}$, the opposite trend is found. Here, a $4 f^{8} 5 d^{1} \underline{L}$ configuration is formed following a S $3 p \rightarrow$ $\mathrm{Tb} 4 f$ virtual excitation. Spin coupling according to $S_{4 f^{8} 5 d^{1}} \times S_{L}$ leads to $\frac{7}{2} \times \frac{1}{2}$, which produces $S=4,3$, and $\frac{5}{2} \times \frac{1}{2}$, which produces $S=3,2$ (see Fig. 4). Then, there is a larger number of $S=3$ virtual excitations than $S=4$ ones, which results in an extra stabilization of the LS $(S=3)$ states with respect to the HS $(S=4)$ states and a corresponding lowering of the exchange splitting. And this effect, of course, is larger for more covalent ligands (see Fig. 4). 


\subsubsection{Excited state bond lengths}

It is interesting to have a close look on how the bond length changes across the manifold of excited states and on the insight it provides on their nature. Equilibrium bond lengths and energies obtained by fitting a third order polynomial to the potential energy curves are displayed in Fig. 5 for $\mathrm{CaS}: \mathrm{Eu}^{2+}$ (see also Table 1 and Electronic Supplementary Information for more data).

It is observed that the levels of the lowest $4 f^{6} 5 d^{1}$ group, i.e. $4 f^{6}\left({ }^{7} F\right) 5 d t_{2 g}^{1}$ in the sulfides and $4 f^{6}\left({ }^{7} F\right) 5 d e_{g}^{1}$ in the fluorides, where the respective most stable $5 d$ molecular orbital shell is occupied, have a shorter bond length than the $4 f^{7}$ ground state. When the levels have the most unstable $5 d$ shell occupied, their bond lengths are longer than in $4 f^{7}$ ground state, e.g. $4 f^{6}\left({ }^{7} F\right) 5 d e_{g}^{1}$ in sulfides and $4 f^{6}\left({ }^{7} F\right) 5 d t_{2 g}^{1}$ in fluorides,

This result is in contrast with many intuitively constructed configurational coordinate models that can be found in literature, where all levels of the $\mathrm{Eu}^{2+} 4 f^{6} 5 d^{1}$ manifold are almost invariably drawn at longer equilibrium distances than $4 f^{7}$ levels, inspired by the fact that $5 d$ orbitals have larger orbital radii than $4 f$ orbitals and reach further. There is a misconception behind this which lies in the implicit assumption that $4 f$ electrons play an important role in the Eu-ligand bond, hence determining the Eu-ligand bond length.

A detailed constrained space orbital variation (CSOV) quantum chemical analysis $\frac{105}{10}$ has shown that the bond length between an $f$-element ion in a $4 f^{N}$ configuration and ligands is realized by the interaction between the $5 p^{6}$ shell and the ligand's valence electrons. When the ion is in a $4 f^{N-1} 5 d$ configuration, additional covalent interactions appear (mostly electron transfer from the ligand to the inner $4 f$ hole) that shorten the bond length; the shortening is enhanced by the $5 d$ ligand field stabilization in $4 f^{N-1} 5 d_{\text {lowest }}^{1}$ configurations and opposed in $4 f^{N-1} 5 d_{\text {highest }}^{1}$ configurations. The magnitudes of these effects result in a systematic bond length trend: $4 f^{N-1} 5 d_{\text {lowest }}^{1}<4 f^{N}$ $<4 f^{N-1} 5 d_{\text {highest }}^{1}$, the differences being smaller than those between $4 f$ and $5 d$ orbital radii. $\frac{105}{105}$ This observation was found in $\mathrm{Ce}^{3+}, \mathrm{Pr}^{3+}, \mathrm{Sm}^{2+}, \mathrm{Tb}^{3+}, \mathrm{Tm}^{2+}$, $\mathrm{Yb}^{3+}$, and $\mathrm{Yb}^{2+}$ as dopants in ionic solids and regarded as generally valid. ${ }^{54}$ Here we confirm the observation for $\mathrm{Eu}^{2+}$ in the difluorides, but also in the more covalent sulfides.

The prediction of a bond length shortening upon the lowest $4 f \rightarrow 5 d$ excitation was shown to imply its redshift under pressure, $\frac{106}{}$ which opened the door for its indirect experimental validation. This effect was in fact demonstrated by Valiente et al. ${ }^{107}$ in high-pressure spectroscopic experiments on $\mathrm{Cs}_{2} \mathrm{NaLuCl}_{6}: \mathrm{Ce}^{3+}$. Multiple similar experiments are reported that show the same effect in $\mathrm{Eu}^{2+}$-doped $\mathrm{MF}_{2}{ }^{108 \mid 109}$ and $\mathrm{CaS}^{110}$ hosts, hence supporting our findings.

Fig. 5 shows that a pure $t_{2 g}$ configurational character for the $5 d$ electron brings about a $0.029 \AA$ bond length shortening with respect to the ground state in

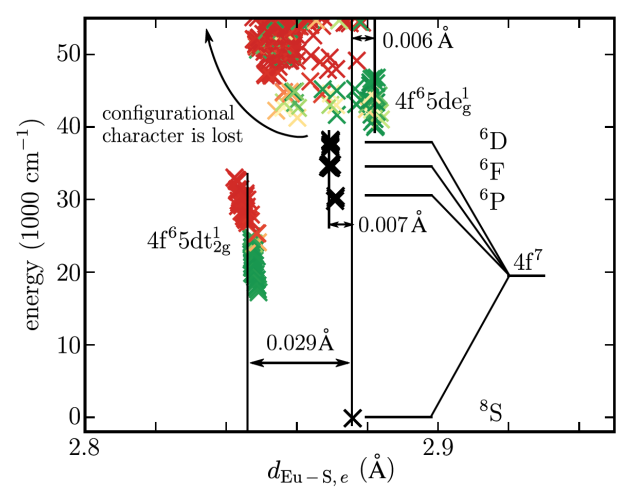

Figure 5 - Equilibrium bond lengths for all potential energy curves of $\mathrm{CaS}: \mathrm{Eu}^{2+}$. This graph indicates the decrease (increase) in bond length for $4 f^{6} 5 d t_{2 q}^{1}$ $\left(4 f^{6} 5 d e_{g}^{1}\right)$ levels with respect to the $4 f^{7}$ manifold. $4 f^{7}$ levels are shown in black crosses. $4 f^{6}(5 d, 6 s)^{1}$ are shown in colored crosses that depend on their $2 S+1$ character at $d_{\mathrm{Eu}-\mathrm{S}}=2.85 \AA$ : $\operatorname{red}(6)$-yellow(mixed)green(8) (see also Fig. 3 .

CaS: $\mathrm{Eu}^{2+}$ and a pure $e_{g}$ character implies a $0.006 \AA$ bond length elongation. However, above approximately $40000 \mathrm{~cm}^{-1}$ the pure configurational character is lost and the mixed $t_{2 g^{-}} e_{g}$ character (as well as $4 f^{6}\left({ }^{7} F\right)-4 f^{6}\left({ }^{5} D\right)$ character $)$ in the eigenstates is translated into intermediate bond lengths. It is clear that the $5 d t_{2 g}$ or $5 d e_{g}$ characters of the wave function determine the excited state bond lengths and that the knowledge of the bond length evolution, e.g. from highpressure spectroscopy, can be used to extract qualitative information about the nature of the wave function.

\section{4 $\mathbf{E u}^{2+} 4 f^{7} \rightarrow 4 f^{6} 5 d^{1}$ spectra}

Along with the calculation of the eigenenergies and wave functions of the studied systems, probabilities for electric dipole transitions are obtained in the form of oscillator strengths(Tables S9 to S14). This enables a direct calculation of absorption or excitation spectra for the $4 f^{7} \rightarrow 4 f^{6} 5 d^{1}$ transitions which can be compared to experimental results from luminescence and UV-VIS spectroscopy. The computed spectra are displayed in Figs. 6 and 7 for the fluorides and sulfides respectively, along with the available experimental spec-

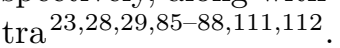

\subsubsection{Spectral assignments}

The computed and experimental spectra are very similar, both in terms of energy ranges as in terms of spectral shape (Figs. 6 and 7). Two broad bands are found in all cases. The first band originates from the spinallowed transitions from the $4 f^{7}\left({ }^{8} S\right)$ ground state to the HS $4 f^{6} 5 d_{\text {lowest }}^{1}$ submanifold. The second band originates from excitations towards the configurationally mixed third and fourth submanifolds. Regardless of the configurational mixing, the good agreement with experiment for the fluorides (where the contributions of LS $4 f^{6} 5 d_{\text {highest }}^{1}$ was not accounted for, see Section 2 

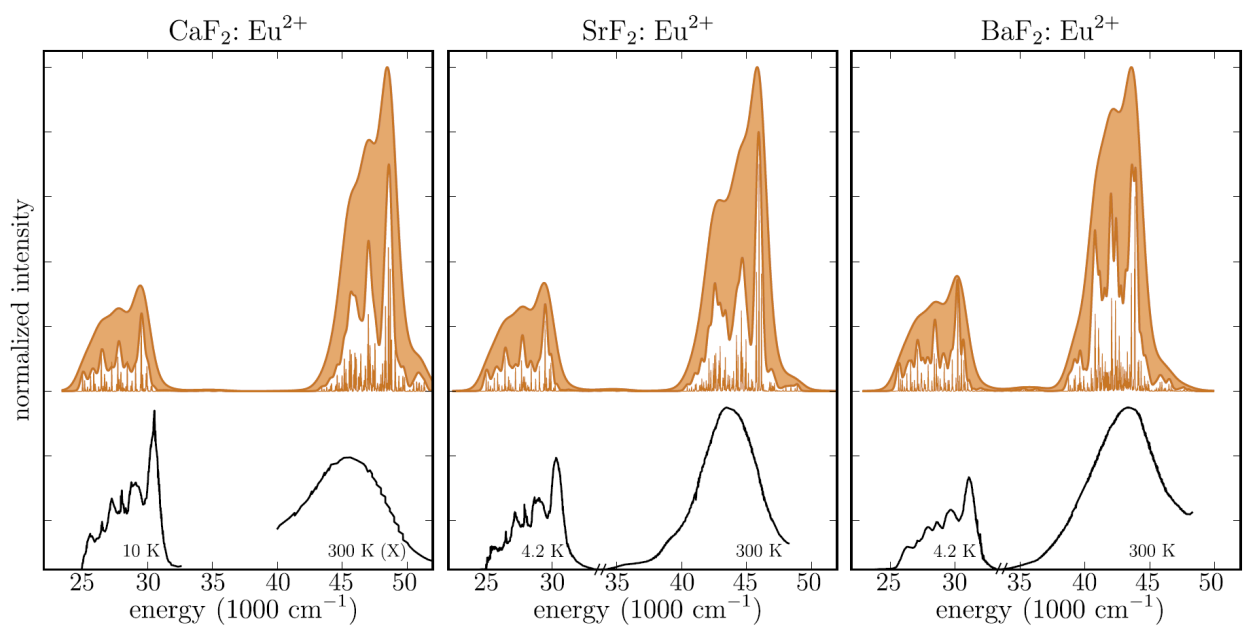

Figure 6 - Top: computed $4 f^{7} \rightarrow 4 f^{6} 5 d^{1}$ absorption spectrum for $\mathrm{Eu}^{2+}$ in the alkaline earth fluorides rendered with broadening parameters of 5,100 and $300 \mathrm{~cm}^{-1}$ and scaled for improved visibility. Bottom: experimental absorption (or excitation, indicated by ' $\mathrm{X}$ ') spectra for the same transitions adapted from Refs. 88 111 (CaF 2$)$, Refs. 28 $85\left(\mathrm{SrF}_{2}\right)$ and Refs. 28185 $\left(\mathrm{BaF}_{2}\right)$. The temperatures at which the experimental spectra were obtained are indicated.
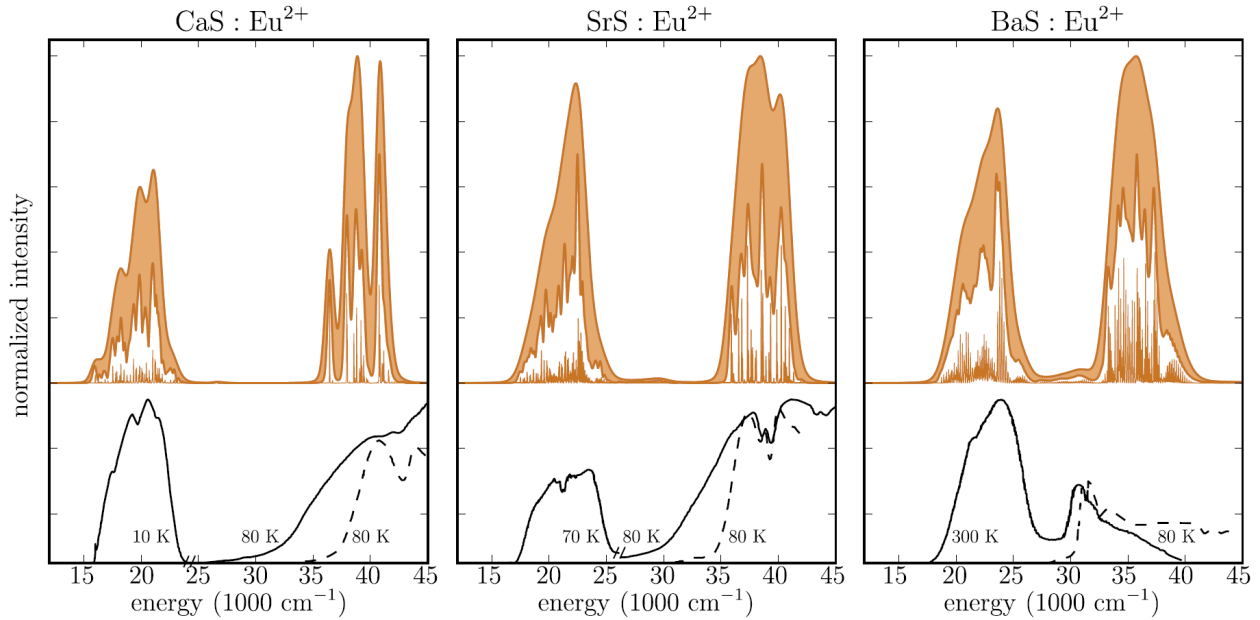

Figure 7 - Top: computed $4 f^{7} \rightarrow 4 f^{6} 5 d^{1}$ absorption spectrum for $\mathrm{Eu}^{2+}$ in the alkaline earth sulfides rendered with broadening parameters of 5, 100 and $300 \mathrm{~cm}^{-1}$. Bottom: experimental excitation spectra for the same transitions adapted from Refs. $86 \mid 87$ (CaS), Refs. 23 87] (SrS) and Ref. 29] (BaS, here the anomalous emission was monitored). The host absorption is also added (dashed lines) to distinct between intrinsic and $\mathrm{Eu}^{2+}$ caused spectral features, adapted from Ref. 112, The temperatures at which the experimental spectra were obtained are indicated. 
and Fig. 3 shows that the second band is dominated by the HS contributions.

Excitation from the $4 f^{7}\left({ }^{8} S\right)$ ground state towards the HS $4 f^{6} 5 d_{\text {lowest }}^{1}$ submanifold is spin-forbidden, yielding a gap between the two bands in the spectrum that is larger than the gap in the associated energy levels schemes (Fig. 3). Even though they are spectroscopically invisible, the presence of the LS levels are important to understand the behavior of $\mathrm{Eu}^{2+}$ based luminescent materials. The LS levels provide an efficient nonradiative relaxation channel, precluding any radiative decay other than that from the lowest level of the $4 f^{6} 5 d^{1}$ manifold.

The gap between the LS $4 f^{6} 5 d_{\text {lowest }}^{1}$ and HS $4 f^{6} 5 d_{\text {highest }}^{1}$ submanifolds becomes smaller when going from the Ca-compounds to the Sr-compounds and almost disappears for the Ba-compounds (see Fig. 3). Therefore, spin-orbit interactions between both submanifolds become larger and the amount of spinmixing is increased, with an average HS content ranging from $0.5 \%$ for $\mathrm{CaF}_{2}$ to $2 \%$ for $\mathrm{BaS}$ for the LS $4 f^{6} 5 d_{\text {lowest }}^{1}$ submanifold. This leads to so-called spinenabled transitions towards these states, which feature a small but observable transition intensity (see Fig. 6 and 7p. Similar spin-enabled transitions were assigned by Suta and Wickleder ${ }^{27}$ for an extraordinarily well-resolved low-temperature absorption spectrum of $\mathrm{SrCl}_{2}: \mathrm{Eu}^{2+}$ single crystals by Karbowiak and Rudowicz 97 .

Interpretation of the spectra of the $\mathrm{Eu}^{2+}$ doped sulfides poses an additional difficulty with respect to the fluorides. Due to their small band gaps, the sulfide hosts already absorb at energies where absorption or photoluminescence spectra are typically measured (see dashed lines in Fig. 7). As a result, the second band in the experimental absorption and excitation spectra of the $\mathrm{Eu}^{2+}$-doped sulfides is to be regarded as the added effect of Eu-centered and host-related excitations.

\subsubsection{Origin of the fine structure}

When measured at room temperature, $\mathrm{Eu}^{2+}$ absorption or excitation spectra look broad and featureless. Only when sufficiently cooled, some fine structure might be resolved. This effect is simulated in the calculated spectra by using a variable widening factor in Fig. 6 and 7. Experimentally, a well-resolved fine structure is found for the fluorides, while this is much less the case for the sulfides (e.g. the excitation spectra of $\mathrm{CaF}_{2}$ and $\mathrm{CaS}$ were both measured at 10 $\mathrm{K})$. This can be attributed to the larger difference between the equilibrium bond lengths, $d_{\mathrm{Eu}-\mathrm{X}, \mathrm{e}}\left(4 f^{6} 5 d^{1}\right)-$ $d_{\mathrm{Eu}-\mathrm{X}, \mathrm{e}}\left(4 f^{7}\left({ }^{8} S\right)\right)$, in case of the sulfides $(e . g .0 .027 \AA$ for $\mathrm{CaS}: \mathrm{Eu}^{2+}$ ), compared to the fluorides (e.g. 0.010 $\AA$ for $\mathrm{CaF}_{2}: \mathrm{Eu}^{2+}$ ).

The electronic origin of this fine structure has been the topic of a long-standing debate in literature, which has been largely confined to empirical and crystal-field theoretical approaches $\sqrt{17+26}$. We address now the origin of the fine structure with the help of crystal field theory calculations performed (with an in-house written
Python code $\mathrm{e}^{(113}$ ) at the light of the ab initio electronic structure summarized in Fig. 3 .

It is common to explain the fine structure in the lowest part of the $\mathrm{Eu}^{2+}$ excitation or absorption spectra with the decoupled scheme, a model described by Freiser, Methfess and Holtzberg in 1968.17 According to it, in the first place, the lowest $4 f^{6} 5 d^{1}$ states can be obtained from the multiplets of the $4 f^{6}\left({ }^{7} F\right)$ core, reminiscent of the lowest $5000 \mathrm{~cm}^{-1}$ of the Dieke diagram for $\mathrm{Eu}^{3+}$, and the lowest crystal-field level of the single $5 d$ electron, which acquires a well-defined $t_{2 g}$ character in the sulfides $\left(e_{g}\right.$ in the difluorides; for simplicity, the discussion is continued for sulfides). This point of view is supported by the multiconfigurational $a b$ initio calculations, as we discussed in Sec. 3.3.2. In the second place, the decoupled scheme assumes that the coupling between the $4 f^{6}\left({ }^{7} F\right)$ core and the $5 d t_{2 g}$ electron is of a mean-field type, i.e. that it does not lead to relevant splittings. Then, since the spin-orbit coupling is very small in the $5 d t_{2 g}$ shell but strong within the $4 f^{6}\left({ }^{7} F\right)$ core, and the $4 f$ electrons are shielded from ligand field effects, the fine structure is entirely due to the $4 f^{6}\left({ }^{7} F\right)$ internal spin-orbit coupling, i.e. the well-known ${ }^{7} F_{J=0-6}$ seven multiplets of $\mathrm{Eu}^{3+}$ (see left panel of Fig. 8).

The decoupled scheme is usually justified by the occurrence of a so-called staircase structure that can be resolved in certain low temperature $\mathrm{Eu}^{2+}$ spectra $\frac{17|20| 21|23| 26}{26}$ and the seven most prominent maxima in the absorption or excitation spectra are labeled as $J=0-6$. However, as we discussed above, the staircase is also found in the ab initio calculations, although the couplings between the $4 f^{6}\left({ }^{7} \mathrm{~F}\right)$ core and the $5 d t_{2 g}$ electron are included and nothing indicates that they are small.

In order to investigate the strength of the $4 f^{6}\left({ }^{7} F\right) \times$ $5 d t_{2 g}$ coupling, hence the validity of the decoupled scheme, the Coulomb interaction between the $4 f$ and $5 d$ electron can be taken into account on crystal field theory level by tuning the value of the Slater-Condon integrals $F^{k}(4 f, 5 d)(k=2,4)$ and $G^{k}(4 f, 5 d)(k=$ $1,3,5)$. This was firstly done by Yanase and Kasuya in 1970 in order to study the optical and magnetic properties of $\mathrm{EuF}_{2}$ and subsequently studied in a more general approach by Weakliem in 1972 on a set of $4 f^{6}\left({ }^{7} F\right) 5 d e_{g}$ levels $18 \mid 19$. Here, we perform a similar simulation on the entire $4 f^{6}\left({ }^{7} F\right) 5 d^{1}$ manifold and compare it to our $a b$ initio excited state landscape. Fig. 8 shows how the eigenvalues of an intermediate coupling calculation for the $4 f^{6}\left({ }^{7} F\right) 5 d^{1}$ manifold evolve with the coupling parameter $G^{1}(4 f, 5 d) / \zeta_{4 f}$, from the decoupled scheme, where the states are labeled as $4 f^{6}\left({ }^{7} F_{J}\right) 5 d \gamma^{1}$ $\left(J=0-6, \gamma=t_{2 g}, e_{g}\right)$, towards the other limiting case, where only the term splitting due to $f-d$ Coulomb interaction and $5 d$ crystal field are accounted for (right panel). Qualitative inspection of this diagram shows that a decoupled scheme can never give a good representation of reality because it shows no overall exchange splitting, which is clearly present in the $a b$ initio excited state landscapes (Fig. 3). The total angular momentum of the $4 f^{6}$ subshell, $J$, is hence not a good 


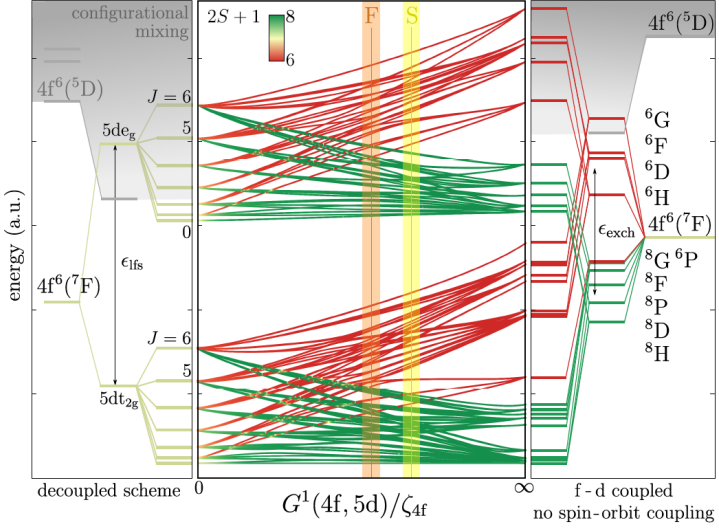

Figure 8 - Crystal field calculation for the low energy part of the $4 f^{6} 5 d^{1}$ configuration in an octahedral field, obtained in the 490 dimensional $\left|4 f^{6}\left({ }^{7} F\right) 5 d^{1}\right\rangle$ basis. The transition from the decoupled scheme (including only $4 f$ spin-orbit coupling as additional interaction) to a scheme with only the $4 f-5 d$ Coulomb interaction is shown. The colors of the curves represent the spin-characters of the eigenstates, ranging from green for pure spin-octets to red for pure spin-sextets. The shaded area indicates the energy range where configurational mixing can be expected (see \$3.3). The vertical bands, denoted by ' $\mathrm{F}$ ' and 'S', denote the parameter ratio where the crystal field calculation resembles best to the $a b$ initio calculations for the fluorides and sulfides, respectively.

quantum number, not even approximately in cases of alkaline earth fluorides or sulfides. The $4 f-5 d$ coupling strengths, as measured with the $G^{1}(4 f, 5 d) / \zeta_{4 f}$ ratios, that give the best qualitative correspondence to the electronic structure of the $\mathrm{Eu}^{2+}$ doped fluorides and sulfides are indicated in Fig. 8 .

Rarely, the electronic structure of the $4 f^{6} 5 d^{1}$ configuration is even more simplified by accounting solely the $5 d$ crystal field splitting, leading to a maximum of five levels for the lowest symmetries ${ }^{42}$. It is then argued that the remaining splittings are negligible with respect to the $5 d$ crystal field splitting and lead at most to a broadening of the spectral features. The above analysis shows however that this is a too severe simplification, overlooking the strong term- and multiplet splitting of the $4 f^{6}$ subshell, the $4 \mathrm{f}-5 \mathrm{~d}$ coupling and the configurational mixing of the $5 d$ wavefunctions.

In summary, the simplified models have been and are still very useful to aid the qualitative interpretation of $\mathrm{Eu}^{2+}$ spectra, even though they fall short for quantitative analysis. If information beyond the low-energy tail of the $4 f^{7} \rightarrow 4 f^{6} 5 d^{1}$ absorption or excitation spectra is however required, also the qualitative similarity to reality will cease. From the experimental point of view, the limited window where these approximations are reasonable, together with the extremely high density of energy levels prohibits the reliable determination of radial integrals such as crystal field parameters, Slater-Condon integrals or spin-orbit constants. Here, $a b$ initio calculations are compulsory.

\subsection{The $\mathbf{E u}^{3+} 4 f^{6}$ configuration}

Trivalent europium, $\mathrm{Eu}^{3+}$, is a lanthanide ion with a rich history in scientific and technological applications. It features a characteristic red or orange emission, underlying its successful application in fluorescent lighting 1144. Furthermore, the low $J$-values of the multiplets that take part in the luminescent transitions make this ion suited as a symmetry probe $\mathrm{e}^{115,[119}$. Notwithstanding the successes of $\mathrm{Eu}^{3+}$, a satisfying analysis of its spectrum is not always possible, due to too small crystal field splittings, which is especially troublesome in case of high coordination numbers, low transition strength, or the need for polarized spectra to distinguish between different point symmetries. In the last case, single crystals are required, which are in practice not always accessible. These shortcomings indicate that the empirical approach would serve well with the support of $a b$ initio calculations.

Here, the spin-septet and spin-quintet states of $\mathrm{Eu}^{3+}$ were calculated upon doping it into the alkaline fluorides and sulfides. As an example, the resulting curves after spin-orbit splitting for $\mathrm{CaF}_{2}: \mathrm{Eu}^{3+}$ are shown in Fig. 9. In all cases, the $\mathrm{Eu}^{3+}$ ion is substituted on a divalent alkaline earth site, requiring the compensation of a singly positive charge. In these calculations, it is assumed that the compensation is non-local, maintaining the high $O_{h}$ symmetry. Magnetic resonance studies on $\mathrm{CaF}_{2}$, doped with $\mathrm{Eu}^{3+}$ or other trivalent lanthanides showed that this situation can be achieved by a rapid quenching of the crystal during the high-temperature synthesis 120 123. In the sulfides, $\mathrm{Eu}^{3+}$ is more easily reduced to $\mathrm{Eu}^{2+}$ and $\mathrm{Eu}^{3+}$ typically occurs along with $\mathrm{Eu}^{2+}$, or not at all. Magnetic resonance studies on $\mathrm{Ce}^{3+}$ in the sulfides showed that approximately half of the ions are in a high-symmetry site $\left(O_{h}\right)$, while the others show a lower site symmetry due to local charge compensation $124 \mid 125$.

Fig. 9 illustrates that the well-known structure of the $4 f^{6}$ configuration is well-reproduced, showing the ${ }^{6} F_{J}(J=0, \ldots, 6)$ multiplets at low energy, followed by a gap of approximately $12000 \mathrm{~cm}^{-1}$ after which the well-separated ${ }^{5} D_{0},{ }^{5} D_{1},{ }^{5} D_{2}$ and ${ }^{5} D_{3}$ multiplets are found. In this low-energy region $J$-mixing is limited, justifying the use of the atomic quantum number $L$, $S$ and $J$. At higher energies, a dense set of levels is found, originating from ${ }^{5} D_{4},{ }^{5} L,{ }^{5} G$ and higher-excited terms. In this region, $J$-mixing is considerable, and the double group irrep labels are the only valid labels. Even though some gaps of $1000 \mathrm{~cm}^{-1}$ or more are found in the excited state landscape, no multiplet label can be attached to any set of levels (see also Electronic Supplementary Information). As an insight into the spin-free result, the term splitting is also displayed in Fig. 9

In case of $\mathrm{CaF}_{2}$, a detailed account of the energy levels of cubic $\mathrm{Eu}^{3+}$ impurities was provided by Gastev et al. .89 , elegantly exploiting the difference in lifetime of the ${ }^{5} D_{0}$ and ${ }^{5} D_{1}$ emitting levels to separate both emissions, enabling a comparison between the calculated and experimental energies (see Table S15). 


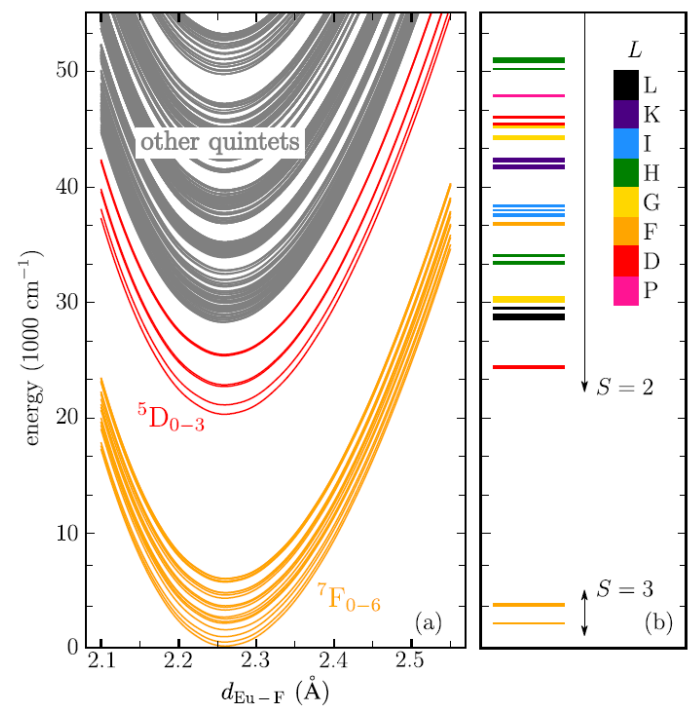

Figure 9 - Potential energy curves for the $4 f^{6}$ configuration of the $\mathrm{Eu}^{3+}$ impurity in $\mathrm{CaF}_{2}$. (a) Result upon inclusion of spin-orbit coupling. (b) Resulting minimum energies of the spin-free calculation, colored according to their dominant ${ }^{2 S+1} L$ character (see Fig. S2 for the corresponding curves).

It is clear that, provided that the same scaling of computed energies, by $90 \%$, is applied, a satisfying correspondence between the experimental and computed levels is found, with deviations of the order of $100 \mathrm{~cm}^{-1}$ (see also red triangles in Fig. 2).

Table 3 summarizes the main results for the $\mathrm{Eu}^{3+}$ calculations in the different hosts, where the energies were averaged over the Stark components of the ${ }^{7} F_{0-6}$ and ${ }^{5} D_{0-3}$ multiplets. It is confirmed that these energies are insensitive to the chemical environment. Yet, an increase of $50-100 \mathrm{~cm}^{-1}$ of the ${ }^{5} D_{0}$ level is found when going from the $\mathrm{Ca}$ to the $\mathrm{Sr}$ and $\mathrm{Ba}$ compounds, and a depression of the same order of magnitude is found when going from fluorides to sulfides. Translated to wavelengths, this corresponds to a shift of at most a few nanometers. Low temperatures and accurately (wavelength) calibrated detectors are hence indispensable to acquire spectroscopic data on the $4 f^{6}$ configuration.

Systematic studies of $\mathrm{Eu}^{3+}$ spectra are often restricted to the energy of the ${ }^{7} F_{0^{-}}{ }^{5} D_{0}$ line which changes over maximally a few $100 \mathrm{~cm}^{-1}$ between different hosts. $115 \mid 126] 129$. Its intensity is especially sensitive to the site symmetry and vanishes when the $\mathrm{Eu}^{3+}$ occupies an inversion center 116 . In that regard, the differences in calculated ${ }^{7} F_{0^{-}}{ }^{5} D_{0}$ energies along the series of Ca-Sr-Ba-compounds and F-S ligands (see Tab. 3) are significant. Three effects play a role: the difference in Eu-ligand bond length along the series Ca-Sr-Ba causes a blueshift, while the decrease in coordination number and the increased nephelauxetic effect when going from fluorides to sulfides cause a redshift.

Interestingly, the equilibrium Eu- $\mathrm{X}(\mathrm{X}=\mathrm{F}, \mathrm{S})$ distance difference between $\mathrm{Eu}^{2+}$ and $\mathrm{Eu}^{3+}, \Delta d_{e}(\mathrm{Eu})$ increases when going from the $\mathrm{Ca}$ to the Ba compound,
Table 3 - Computed equilibrium Eu-F and Eu-S distances (in $\AA$ ), ground state breathing mode vibrational frequencies (in $\mathrm{cm}^{-1}$ ), and average scaled energies for the lowest multiplets (in $\mathrm{cm}^{-1}$ ) of $\mathrm{Eu}^{3+}$ in $\mathrm{MF}_{2}$ and $\mathrm{MS}(\mathrm{M}=\mathrm{Ca}, \mathrm{Sr}, \mathrm{Ba}) . \mathrm{X}=\mathrm{F}, \mathrm{S}$.

\begin{tabular}{rrrrrrr} 
& $\mathrm{CaF}_{2}$ & $\mathrm{SrF}_{2}$ & $\mathrm{BaF}_{2}$ & $\mathrm{CaS}$ & $\mathrm{SrS}$ & $\mathrm{BaS}$ \\
\hline$d_{\mathrm{Eu}-\mathrm{X}, e}$ & 2.261 & 2.313 & 2.363 & 2.729 & 2.800 & 2.829 \\
$\nu_{e}$ & 495 & 445 & 402 & 307 & 286 & 248 \\
& \multicolumn{5}{c}{$4 f^{6}\left({ }^{7} F_{J}\right)$} \\
$J=0$ & 0 & 0 & 0 & 0 & 0 & 0 \\
1 & 311 & 323 & 327 & 335 & 336 & 338 \\
2 & 940 & 949 & 954 & 957 & 961 & 963 \\
3 & 1877 & 1850 & 1844 & 1831 & 1826 & 1825 \\
4 & 2887 & 2849 & 2836 & 2803 & 2797 & 2796 \\
5 & 3978 & 3932 & 3909 & 3839 & 3839 & 3839 \\
6 & 5120 & 5068 & 5047 & 4991 & 4980 & 4976 \\
& & & $4 f^{6}\left({ }^{5} D_{J}\right)$ & & \\
$J=0$ & 18021 & 18025 & 18075 & 17904 & 17976 & 18007 \\
1 & 18745 & 18752 & 18804 & 18629 & 18703 & 18735 \\
2 & 20255 & 20266 & 20322 & 20140 & 20219 & 20252 \\
3 & 22646 & 22668 & 22732 & 22544 & 22626 & 22663 \\
\hline
\end{tabular}

0.13, 0.16 and $0.20 \AA$ for $\mathrm{MF}_{2}$, while being $0.15,0.18$ and $0.26 \AA$ for $\mathrm{MS}(\mathrm{M}=\mathrm{Ca}, \mathrm{Sr}, \mathrm{Ba})$. This parameter is of major importance to appreciate the effect of charge-transfer states on the Eu-induced luminescence. It was shown that the larger $\Delta d_{e}(\mathrm{Eu})$ for $\mathrm{BaF}_{2}$ is directly responsible for the complete quenching of the $\mathrm{Eu}^{2+} 4 f^{6} 5 d^{1} \rightarrow 4 f^{6}$ emission by intervalence charge transfer, while this is not the case in $\mathrm{CaF}_{2}$ or $\mathrm{SrF}_{2}{ }^{[130]}$.

The above numbers also indicate that $\Delta d_{e}(\mathrm{Eu})$ is larger for the sulfides than for the fluorides. The reason is that the sulfides are more elastic than the fluorides, and undergo a larger "strain", characterized by $\Delta d_{e}(\mathrm{Eu})$ upon the changing chemical "stress", as quantified by the bulk moduli $131 \mid 132$.

\section{Conclusions}

Multiconfigurational $a b$ initio embedded cluster calculations have been performed on the energies and wave functions of the crowded $4 f^{7}$ and $4 f^{6} 5 d^{1}$ manifolds of $\mathrm{Eu}^{2+}$ and the $4 f^{6}$ manifold of $\mathrm{Eu}^{3+}$ doped into two types of chemically different host crystals, the alkaline earth difluorides $\mathrm{MF}_{2}$ and the more covalent alkaline earth sulfides $\mathrm{MS}(\mathrm{M}=\mathrm{Ca}, \mathrm{Sr}, \mathrm{Ba})$. Handling the numerous, highly correlated and dense sets of excited states made the theoretical methodology face its highest level of complexity so far. The excited states have been identified and analyzed, and their configuration coordinate diagrams calculated, which allowed to extract excited state bond lengths and breathing mode vibrational frequencies. This, together with the calculation of electric dipole transition moments and oscillator strengths, allowed for the production of theoretical $4 f \rightarrow 5 d$ spectral profiles directly comparable with experiments. The agreement is excellent.

All computed quantities were compared to experimental data when available. The calculated energy level locations show a systematic overestimation of 
$10 \%$ due to the used approximations and truncations that keep the calculation manageable. After correction for this systematic error, a quantitative agreement is found between computed and spectroscopically determined level locations with a $68 \%$ prediction interval of $300 \mathrm{~cm}^{-1}$.

Calculated Eu-F and Eu-S equilibrium distances for ground and excited states indicate that the lowest $4 f \rightarrow 5 d$ excitation $\left(4 f \rightarrow 5 d e_{g}\right.$ in difluorides and $4 f \rightarrow 5 d t_{2 g}$ in sulfides) leads to a compression of the coordination polyhedron of $\mathrm{Eu}^{2+}$, which explains the red shift of these transitions under high-pressure. Excitation to the highest $5 d$ shell $\left(5 d t_{2 g}\right.$ in difluorides, $5 d e_{g}$ in sulfides) leads to an expansion, but this holds for a limited number of states only, because at the energies of these excitations severe configurational mixing exists that leads to intermediate bond lengths.

The $4 f-5 d$ exchange splittings in the $4 f^{6} 5 d^{1}$ configurations of $\mathrm{Eu}^{2+}$ was found to be $150 \%$ larger in the sulfides than in the difluorides. This behavior, which is opposite to the $4 f^{7} 5 d^{1}$ configurations of $\mathrm{Tb}^{3+}$, was explained on the basis of covalency, which enhances the role of ligand-to- $4 f$ virtual excitations, which over stabilize high-spin states in $\mathrm{Eu}^{2+}$ and low-spin states in $\mathrm{Tb}^{3+}$.

The fine structure of the $4 f \rightarrow 5 d$ absorption and excitation spectra, the so-called staircase structure, was well reproduced in the $a b$ initio calculations. A detailed investigation of the strength of the $4 f^{6}\left({ }^{7} F\right) \times 5 d t_{2 g}$ coupling in sulfides via crystal-field-theory calculations revealed that the popular "decoupled scheme", which attributes the fine structure to the $J=0-6$ levels of the $4 f^{6}\left({ }^{7} F_{J}\right)$ subshell, is not justified. An intermediate coupling is compulsory and it is the interplay between the spin-orbit coupling within the $4 f^{6}\left({ }^{7} F_{J}\right)$ subshell and the $4 f-5 d$ exchange splitting, i.e. the splitting into a high-spin and low-spin part, what dominates the fine structures of the $4 f^{6}\left({ }^{7} F\right) 5 d t_{2 g}^{1}$ and $4 f^{6}\left({ }^{7} F\right) 5 d e_{g}^{1}$ sets.

Overall, these multiconfigurational ab initio calculations offer a detailed view on the excited state landscape of Eu-doped solids which underlies their optical and luminescence properties. The ability to get an accurate description of very different host crystals opens new doors to use this approach to help designing new functional materials for technological applications where requirements are very restrictive and to answer fundamental questions related to Eu-doped phosphors.

\section{Conflicts of interest}

There are no conflicts to declare.

\section{Acknowledgements}

J.J.J. acknowledges the UGent Special Research Fund (BOF/PDO/ 2017/002101) and the Fund for Scientific Research-Flanders (FWO) for a travel grant (V416818N). This work was partially supported by
Ministerio de Economía y Competitividad, Spain (Dirección General de Investigación y Gestión del Plan Nacional de I+D+i, MAT2017-83553-P).

\section{References}

[1] P. F. Smet, A. B. Parmentier and D. Poelman, Selecting Conversion Phosphors for White LightEmitting Diodes, J. Electrochem. Soc., 2011, 158, R37-R54.

[2] S. Abe, J. J. Joos, L. I. D. J. Martin, Z. Hens and P. F. Smet, Hybrid Remote Quantum Dot/Powder Phosphor Designs for Display Backlights, Light: Sci. Appl., 2017, 6, e16271.

[3] B. V. Shulg'in, S. I. Buzmakova, L. V. Viktorov, A. L. Krymov, V. L. Petrov, S. V. Podurovskii, A. A. Kozlov, B. M. Shapiro, M. Y. Shrom, A. I. Nepomnyashchikh, P. V. Figura and V. M. Lakhov, Scintillation Detectors Working with $\mathrm{CaF}_{2}-\mathrm{Eu}$ Single-Crystals, Atom. Energy, 1993, 75, 534-538.

[4] P. Leblans, D. Vandenbroucke and P. Willems, Storage Phosphors for Medical Imaging, Materials, 2011, 4, 1034-1086.

[5] P. Pust, V. Weiler, C. Hecht, A. Tücks, A. S. Wochnik, A. K. Henß, D. Wiechert, C. Scheu, P. J. Schmidt and W. Schnick, Narrow-Band Red-Emitting $\operatorname{Sr}\left[\mathrm{LiAl}_{3} \mathrm{~N}_{4}\right]: \mathrm{Eu}^{2+}$ as a Next-Generation LED-Phosphor Material, Nat. Mater., 2014, 13, 891-896.

[6] X. Qin, X. W. Liu, W. Huang, M. Bettinelli and X. G. Liu, Lanthanide-Activated Phosphors Based on 4f-5d Optical Transitions: Theoretical and Experimental Aspects, Chem. Rev., 2017, 117, 4488-4527.

[7] M. Zhao, H. Liao, L. Ning, Q. Zhang, Q. Liu and Z. Xia, Next-Generation Narrow-Band GreenEmitting RbLi(Li3SiO4)2:Eu2+ Phosphor for Backlight Display Application, Adv. Mater., 2018, 30, 1802489.

[8] M. Zhao, H. X. Liao, M. S. Molokeev, Y. Y. Zhou, Q. Y. Zhang, Q. L. Liu and Z. G. Xia, Emerging Ultra-Narrow-Band CyanEmitting Phosphor for white LEDs with Enhanced Color Rendition, Light: Sci. Appl., 2019, $\mathbf{8}, 38$.

[9] J. Qiao, G. Zhou, Y. Zhou, Q. Zhang and Z. Xia, Divalent Europium-Doped Near-InfraredEmitting Phosphor for Light-Emitting Diodes, Nat. Commun., 2019, 10, 5267.

[10] C. K. Jørgensen, Electron Transfer Spectra of Lanthanide Complexes, Mol. Phys., 1962, 5, 271-277. 
[11] J. L. Ryan and C. K. Jørgensen, Absorption Spectra of Octahedral Lanthanide Hexahalides, J. Phys. Chem., 1966, 70, 2845-2857.

[12] M. J. J. Lammers and G. Blasse, Luminescence of $\mathrm{Tb}^{3+}$-Activated and $\mathrm{Ce}^{3+}$-Activated Rare-Earth Silicates, J. Electrochem. Soc., 1987, 134, 20682072 .

[13] V. Bachmann, C. Ronda, O. Oeckler, W. Schnick and A. Meijerink, Color Point Tuning for ( $\mathrm{Sr}, \mathrm{Ca}, \mathrm{Ba}) \mathrm{Si}_{2} \mathrm{O}_{2} \mathrm{~N}_{2}: \mathrm{Eu}^{2+}$ for White Light LEDs, Chem. Mater., 2009, 21, 316-325.

[14] Z. Xia and A. Meijerink, Ce ${ }^{3+}$-Doped Garnet Phosphors: Composition Modification, Luminescence Properties and Applications, Chem. Soc. Rev., 2017, 46, 275-299.

[15] J. J. Joos, D. Poelman and P. F. Smet, Energy Level Modeling of Lanthanide Materials: Review and Uncertainty Analysis, Phys. Chem. Chem. Phys., 2015, 17, 19058-19078.

[16] International Telecommunication Union, Parameter Values for Ultra-High Definition Television Systems for Production and International Programme Exchange, Recommendation ITU-R BT2020, 2015, 1, 1-8.

[17] M. J. Freiser, S. Methfess and F. Holtzber, Multiplet Structure in Absorption Spectrum of $\mathrm{Eu}^{2+}$, J. Appl. Phys., 1968, 39, 900-902.

[18] A. Yanase and T. Kasuya, Magneto-Optical Effect Due to $\mathrm{Eu}^{2+}$ Ion, Progress of Theoretical Physics, 1970, 46, 388-410.

[19] H. A. Weakliem, Electronic Interactions in $4 \mathrm{f}^{6} 5 \mathrm{~d}$ Configuration of $\mathrm{Eu}^{2+}$ in Crystals, Phys. Rev. B, 1972, 6, 2743-2748.

[20] F. M. Ryan, W. Lehmann, D. W. Feldman and J. Murphy, Fine-Structure in Optical-Spectra of Divalent Europium in Alkaline-Earth Sulfates, $J$. Electrochem. Soc., 1974, 121, 1475-1481.

[21] A. Meijerink and G. Blasse, Luminescence Properties of $\mathrm{Eu}^{2+}$-Activated Alkaline-Earth Haloborates, J. Lumin., 1989, 43, 283-289.

[22] Z. F. Pan, L. X. Ning, B. M. Cheng and P. A. Tanner, Absorption, Excitation and Emission Spectra of $\mathrm{SrCl}_{2}: \mathrm{Eu}^{2+}$, Chem. Phys. Lett., 2006, 428, 78-82.

[23] J. E. Van Haecke, P. F. Smet and D. Poelman, Luminescent Characterization of $\mathrm{CaAl}_{2} \mathrm{~S}_{4}: \mathrm{Eu}$ Powder, J. Lumin., 2007, 126, 508-514.

[24] M. Suta, P. Larsen, F. Lavoie-Cardinal and C. Wickleder, Photoluminescence of $\mathrm{CsMBr}_{3}: \mathrm{Eu}^{2+}(\mathrm{M}=\mathrm{Mg}, \mathrm{Ca}, \mathrm{Sr})-\mathrm{A}$ Novel Strategy for the Development of Low-Energy Emitting Phosphors, J. Lumin., 2014, 149, 35-44.
[25] S. J. Camardello, P. J. Toscano, M. G. Brik and A. M. Srivastava, Optical Spectroscopy, Thermal Quenching and Electron-Vibrational Interaction of the Octahedrally Coordinated $\mathrm{Eu}^{2+}$ Ion in $\mathrm{SrAl}_{2} \mathrm{~B}_{2} \mathrm{O}_{7}$, J. Lumin., 2014, 151, 256-260.

[26] O. M. ten Kate, T. Vranken, E. van der Kolk, A. P. J. Jansen and H. T. Hintzen, Optical Properties of $\mathrm{Eu}^{2+} / \mathrm{Eu}^{3+}$ Mixed Valence, Silicon Nitride Based Materials, J. Solid State Chem., 2014, 213, 126-131.

[27] M. Suta and C. Wickleder, Synthesis, Spectroscopic Properties and Applications of Divalent Lanthanides Apart from $\mathrm{Eu}^{2+}$, J. Lumin., 2019, 210, 210-238.

[28] B. Moine, C. Pedrini and B. Courtois, Photoionization and Luminescences in $\mathrm{BaF}_{2}-\mathrm{Eu}^{2+}, J$. Lumin., 1991, 50, 31-38.

[29] P. F. Smet, J. E. Van Haecke, F. Loncke, H. Vrielinck, F. Callens and D. Poelman, Anomalous Photoluminescence in BaS:Eu, Phys. Rev. B, 2006, 74, 035207.

[30] D. Dutczak, C. Ronda, T. Justel and A. Meijerink, Anomalous Trapped Exciton and d-f Emission in $\mathrm{Sr}_{4} \mathrm{Al}_{14} \mathrm{O}_{25}: \mathrm{Eu}^{2+}$, J. Phys. Chem. A, 2014, 118, 1617-1621.

[31] Y. F. Liu, C. H. Zhang, Z. X. Cheng, Z. Zhou, J. Jiang and H. C. Jiang, Origin and Luminescence of Anomalous Red-Emitting Center in Rhombohedral $\mathrm{Ba}_{9} \mathrm{Lu}_{2} \mathrm{Si}_{6} \mathrm{O}_{24}: \mathrm{Eu}^{2+}$ Blue Phosphor, Inorg. Chem., 2016, 55, 8628-8635.

[32] J. J. Joos, L. Seijo and Z. Barandiarán, Direct Evidence of Intervalence Charge-Transfer States of Eu-Doped Luminescent Materials, J. Phys. Chem. Lett., 2019, 10, 1581-1586.

[33] K. Van den Eeckhout, P. F. Smet and D. Poelman, Persistent Luminescence in $\mathrm{Eu}^{2+}$-Doped Compounds: A Review, Materials, 2010, 3, 2536-2566.

[34] D. Van der Heggen, J. J. Joos and P. F. Smet, Importance of Evaluating the Intensity Dependency of the Quantum Efficiency: Impact on LEDs and Persistent Phosphors, ACS Photonics, 2018, 5, 4529-4537.

[35] J. Xu and S. Tanabe, Persistent Luminescence Instead of Phosphorescence: History, Mechanism, and Perspective, J. Lumin., 2019, 205, 581-620.

[36] C. K. Duan and M. F. Reid, A Simple Model for $\mathrm{f} \rightarrow \mathrm{d}$ Transitions of Rare-Earth Ions in Crystals, J. Solid State Chem., 2003, 171, 299-303.

[37] C. K. Duan, S. D. Xia, M. F. Reid and G. Ruan, Study of the $\mathrm{f} \rightarrow \mathrm{d}$ Transition of Heavy Lanthanide and Actinide Ions in Crystals Using the 
Simple Model, Phys. Status Solidi B, 2005, 242, 2503-2508.

[38] C. K. Duan, M. F. Reid and G. Ruan, A Simple Model for the f-d Transition of Actinide and Heavy Lanthanide Ions in Crystals, Curr. Appl. Phys., 2006, 6, 359-362.

[39] C. G. Ma, M. F. Reid, C. K. Duan, S. D. Xia and M. Yin, Recent Advances in Simple Model for $4 \mathrm{f}-5 \mathrm{~d}$ Transitions of Lanthanide Ions in Solids, J. Rare Earth, 2007, 25, 262-267.

[40] B. Z. Malkin, Z. I. Ivanenko and I. B. Aizenberg, author, Fizika Tverdogo Tela, 1970, 12, 1873.

[41] B. Z. Larionov, A. L.; Malkin, Effective Hamiltonian of Valence Electrons of Rare-Earth Elements in Ionic Crystals, Opt. Spectrosc., 1975, 39, 637-639.

[42] M. Nazarov, M. G. Brik, D. Spassky and B. Tsukerblat, Crystal Field Splitting of $5 \mathrm{~d}$ States and Luminescence Mechanism in $\mathrm{SrAl}_{2} \mathrm{O}_{4}: \mathrm{Eu}^{2+}$ Phosphor, J. Lumin., 2017, 182, 79-86.

[43] M. F. Reid, C. K. Duan and H. W. Zhou, CrystalField Parameters from Ab Initio Calculations, $J$. Alloys Compd., 2009, 488, 591-594.

[44] L. S. Hu, M. F. Reid, C. K. Duan, S. D. Xia and M. Yin, Extraction of Crystal-Field Parameters for Lanthanide Ions from QuantumChemical Calculations, J. Phys.: Condens. Matter, 2011, 23, 045501.

[45] H. Ramanantoanina, F. Cimpoesu, C. Göttel, M. Sahnoun, B. Herden, M. Suta, C. Wickleder, W. Urland and C. Daul, Prospecting Lighting Applications with Ligand Field Tools and Density Functional Theory: A First-Principles Account of the $4 \mathrm{f}^{7}-4 \mathrm{f}^{6} 5 \mathrm{~d}^{1}$ Luminescence of $\mathrm{CsMgBr}_{3}: \mathrm{Eu}^{2+}$, Inorg. Chem., 2015, 54, 83198326 .

[46] H. Ramanantoanina, M. Sahnoun, A. Barbiero, M. Ferbinteanu and F. Cimpoesu, Development and Applications of the LFDFT: the Nonempirical Account of Ligand Field and the Simulation of the f-d Transitions by Density Functional Theory, Phys. Chem. Chem. Phys., 2015, 17, 18547-18557.

[47] A. Atanasov, C. A. Daul and C. Rauzy, A DFT Based Ligand Field Theory, Struct. Bonding, 2004, 106, 97-125.

[48] G. Sánchez-Sanz, L. Seijo and Z. Barandiarán, Spin-forbidden and Spin-enabled $4 \mathrm{f}^{14} \rightarrow 4 \mathrm{f}^{13} 5 \mathrm{~d}^{1}$ Transitions of $\mathrm{Yb}^{2+}$-Doped $\mathrm{CsCaBr}_{3}$, J. Chem. Phys., 2009, 131, 12591-12598.
[49] Z. Barandiarán, M. Bettinelli and L. Seijo, Color Control of $\mathrm{Pr}^{3+}$ Luminescence by Electron-Hole Recombination Energy Transfer in $\mathrm{CaTiO}_{3}$ and $\mathrm{CaZrO}_{3}$, J. Phys. Chem. Lett., 2017, 8, 30953100 .

[50] L. Seijo and Z. Barandiarán, Intervalence Charge Transfer Luminescence: The Anomalous Luminescence of Cerium-Doped $\mathrm{Cs}_{2} \mathrm{LiLuCl}_{6}$ Elpasolite, J. Chem. Phys., 2014, 141, 214706.

[51] R. B. Hughes-Currie, K. V. Ivanovskikh, J. P. R. Wells, M. F. Reid, R. A. Gordon, L. Seijo and Z. Barandiarán, X-ray Excitation Triggers Ytterbium Anomalous Emission in $\mathrm{CaF}_{2}: \mathrm{Yb}$ but Not in $\mathrm{SrF}_{2}: \mathrm{Yb}$, J. Phys. Chem. Lett., 2017, 8, 1175 1178 .

[52] C. MacKeen, F. Bridges, L. Seijo, Z. Barandiarán, M. Kozina, A. Mehta, M. F. Reid and J. P. R. Wells, The Complexity of the $\mathrm{CaF}_{2}: \mathrm{Yb}$ System: A Huge, Reversible, X-ray-Induced Valence Reduction, J. Phys. Chem. C, 2017, 121, 28435-28442.

[53] M. Dolg, Computational Methods in Lanthanide and Actinide Chemistry, Wiley, 2015.

[54] L. Seijo and Z. Barandiarán, in Ab Initio Calculations on Excited States of Lanthanide Containing Materials, ed. Bünzli, J. C. and Pecharsky, V. K., Elsevier, 2016, vol. 50, ch. 285, pp. 65-89.

[55] L. Seijo and Z. Barandiarán, Host Effects on the Optically Active 4f and 5d Levels of $\mathrm{Ce}^{3+}$ in Garnets, Phys. Chem. Chem. Phys., 2013, 15, 19221-19231.

[56] L. Seijo and Z. Barandiarán, 4f and 5d Levels of $\mathrm{Ce}^{3+}$ in $D_{2}$ 8-Fold Oxygen Coordination, Opt. Mater., 2013, 35, 1932-1940.

[57] J. L. Pascual, Z. Barandiarán and L. Seijo, Ab Initio Theoretical Study of the $4 \mathrm{f}^{8}$ and $4 \mathrm{f}^{7} 5 \mathrm{~d}$ Manifolds of $\mathrm{Tb}^{3+}$-doped $\mathrm{BaF}_{2}$ Cubic Sites, $J$. Lumin., 2014, 145, 808-817.

[58] M. de Jong, A. Meijerink, L. Seijo and Z. Barandiarán, Energy Level Structure and Multiple $4 \mathrm{f}^{12} 5 \mathrm{~d}^{1}$ Emission Bands for $\mathrm{Tm}^{2+}$ in Halide Perovskites: Theory and Experiment, J. Phys. Chem. C, 2017, 121, 10095-10101.

[59] F. Aiga, R. Hiramatsu and K. Ishida, Ab initio theoretical study of $4 \mathrm{f} \rightarrow 5 \mathrm{~d}$ transitions in $\mathrm{Eu}^{2+}$. doped $\mathrm{CaF}_{2}$, J. Lumin., 2014, 145, 951-955.

[60] L. Ning, X. Huang, Y. Huang and P. A. Tanner, Origin of the Green Persistent Luminescence of Eu-Doped SrAl2O4 from a Multiconfigurational Ab Initio Study of $4 \mathrm{f}^{7} \rightarrow 4 \mathrm{f}^{6} 5 \mathrm{~d}^{1}$ Transitions, $J$. Mater. Chem. C, 2018, 6, 6637-6640. 
[61] Z. Barandiarán and L. Seijo, The Ab Initio Model Potential Representation of the Crystalline Environment. Theoretical Study of the Local Distortion on $\mathrm{NaCl}: \mathrm{Cu}^{+}$, J. Chem. Phys., 1988, 89, 5739-5746.

[62] A. Gellé and M. B. Lepetit, Fast Calculation of the Electrostatic Potential in Ionic Crystals by Direct Summation Method, J. Chem. Phys., 2008, 128, 244716.

[63] P.-A. Malmqvist, A. Rendell and B. O. Roos, The Restricted Active Space Self-ConsistentField Method, Implemented with a Split Graph Unitary-Group Approach, J. Phys. Chem., 1990, 94, 5477-5482.

[64] P.-A. Malmqvist, K. Pierloot, A. R. M. Shahi, C. J. Cramer and L. Gagliardi, The Restricted Active Space Followed by Second-order Perturbation Theory Method: Theory and Application to the Study of $\mathrm{CuO}_{2}$ and $\mathrm{Cu}_{2} \mathrm{O}_{2}$ systems, $J$. Chem. Phys., 2008, 128, 204109.

[65] M. Douglas and N. M. Kroll, Quantum Electrodynamical Corrections to the Fine Structure of Helium, Ann. Phys. (N.Y.), 1974, 82, 89-155.

[66] B. A. Hess, Relativistic Electronic-Structure Calculations Employing a Two-Component No-Pair Formalism with External-Field Projection Operators, Phys. Rev. A, 1986, 33, 3742-3748.

[67] R. Llusar, M. Casarrubios, Z. Barandiarán and L. Seijo, Ab Initio Model Potential Calculations on the Electronic Spectrum of $\mathrm{Ni}^{2+}$-doped $\mathrm{MgO}$ Including Correlation, Spin-Orbit and Embedding Effects, J. Chem. Phys., 1996, 105, 53215330 .

[68] P.-A. Malmqvist, B. O. Roos and B. Schimmelpfennig, The RASSI Approach with SpinOrbit Coupling, Chem. Phys. Lett., 2002, 357, 230-240.

[69] B. O. Roos, P. R. Taylor and P. E. M. Siegbahn, A Complete Active Space SCF Method (CASSCF) Using a Density-Matrix Formulated Super-CI Approach, Chem. Phys., 1980, 48, 157173.

[70] P. E. M. Siegbahn, A. Heiberg, B. O. Roos and B. Levy, Comparison of the Super-CI and the Newton-Raphson Scheme in the Complete Active Space SCF Method, Phys. Scr., 1980, 21, 323327.

[71] P. E. M. Siegbahn, A. Heiberg, J. Almlöf and B. O. Roos, The Complete Active Space SCF (CASSCF) Method in a Newton-Raphson Formulation with Application to the HNO Molecule, J. Chem. Phys., 1981, 74, 2384-2396.
[72] J. Olsen, B. O. Roos, P. Jørgensen and H. J. A. Jensen, Determinant Based ConfigurationInteraction Algorithms for Complete and Restricted Configuration-Interaction Spaces, J. Chem. Phys., 1988, 89, 2185-2192.

[73] K. Andersson, P.-Å. Malmqvist, B. O. Roos, A. J. Sadlej and K. Wolinski, Second-Order Perturbation Theory with a CASSCF Reference Function, J. Phys. Chem., 1990, 94, 5483-5488.

[74] K. Andersson, P.-Å. Malmqvist and B. O. Roos, Second-Order Perturbation Theory with a Complete Active Space Self-Consistent Field Reference Function, J. Chem. Phys., 1992, 96, 1218 1226.

[75] A. Zaitsevskii and J.-P. Malrieu, MultiPartitioning Quasidegenerate Perturbation Theory. A New Approach to Multireference Møller-Plesset Perturbation Theory, Chem. Phys. Lett., 1995, 233, 597-604.

[76] J. Finley, P.-A. Malmqvist, B. O. Roos and L. Serrano-Andrés, The Multi-State CASPT2 Method, Chem. Phys. Lett., 1998, 288, 299-306.

[77] C. Froese-Fischer, Oscillator Strengths for ${ }^{2} \mathrm{~S}-{ }^{2} \mathrm{P}$ Transitions in the Copper Sequence, J. Phys. B, 1977, 10, 1241-1251.

[78] T. H. Dunning, B. H. Botch and J. F. Harrison, On the Orbital Description of the $4 \mathrm{~s} 3 \mathrm{~d}^{n+1}$ States of the Transition-Metal Atoms, J. Chem. Phys., 1980, 72, 3419-3420.

[79] Z. Barandiarán and L. Seijo, Radial Correlation Effects on Interconfigurational Excitations At the End of the Lanthanide Series: A Restricted Active Space Second Order Perturbation Study of $\mathrm{Yb}^{2+}$ and $\mathrm{SrCl}_{2}: \mathrm{Yb}^{2+}$, J. Chem. Phys., 2013, 138, 074102.

[80] G. Sánchez-Sanz, L. Seijo and Z. Barandiarán, $\mathrm{Yb}^{2+}$-doped $\mathrm{SrCl}_{2}$ : Electronic Structure of Impurity States and Impurity-Trapped Excitons, $J$. Chem. Phys., 2010, 133, 114509.

[81] G. Karlström, R. Lindh, P.-Å. Malmqvist, B. O. Roos, U. Ryde, V. Veryazov, P. O. Widmark, M. Cossi, B. Schimmelpfennig, P. Neogrady and L. Seijo, MOLCAS: A Program Package for Computational Chemistry, Comp. Mater. Sci., 2003, 28, 222-239.

[82] B. O. Roos, R. Lindh, P. A. Malmqvist, V. Veryazov and P. O. Widmark, Main Group Atoms and Dimers Studied with a New Relativistic ANO Basis Set, J. Phys. Chem. A, 2004, 108, 2851-2858.

[83] B. O. Roos, R. Lindh, P. A. Malmqvist, V. Veryazov, P. O. Widmark and A. C. Borin, New Relativistic Atomic Natural Orbital Basis Sets for Lanthanide Atoms with Applications to the Ce Diatom and $\mathrm{LuF}_{3}$, J. Phys. Chem. A, 2008, 112, 11431-11435. 
[84] M. C. Downer, C. D. Corderomontalvo and H. Crosswhite, Study of New $4 \mathrm{f}^{7}$ Levels of $\mathrm{Eu}^{2+}$ in $\mathrm{CaF}_{2}$ and $\mathrm{SrF}_{2}$ Using Two-Photon Absorption-Spectroscopy, Phys. Rev. B, 1983, 28, 4931-4943.

[85] C. Dujardin, B. Moine and C. Pedrini, OnePhoton and Two-Photon Spectroscopy of $\mathrm{f} \rightarrow \mathrm{d}$ and $\mathrm{f} \rightarrow \mathrm{f}$ Transitions of $\mathrm{Eu}^{2+}$ Ions in $\mathrm{M}_{1-x} \mathrm{~N}_{x} \mathrm{~F}_{2}$ Mixed Fluoride-Crystals (M, N = Ba, Sr, Ca; $0 \leq x \leq 1)$, J. Lumin., 1993, 54, 259-270.

[86] N. Yamashita, S. Fukumoto, S. Ibuki and H. Ohnishi, Photoluminescence of $\mathrm{Eu}^{2+}$ and $\mathrm{Eu}^{3+}$ Centers in CaS:Eu,Na Phosphors, Jpn. J. Appl. Phys., 1993, 32, 3135-3139.

[87] N. Yamashita, O. Harada and K. Nakamura, Photoluminescence Spectra of $\mathrm{Eu}^{2+}$ Centers in $\mathrm{Ca}(\mathrm{S}, \mathrm{Se}) \mathrm{Eu}$ and $\mathrm{Sr}(\mathrm{S}, \mathrm{Se}) \mathrm{Eu}$, Jpn. J. Appl. Phys., 1995, 34, 5539-5545.

[88] C. Pedrini, M. F. Joubert and D. S. McClure, Photoionization Processes of Rare-Earth Dopant Ions in Ionic Crystals, J. Lumin., 2007, 125, 230237.

[89] S. V. Gastev, J. K. Choi and R. J. Reeves, Laser Spectroscopy of $\mathrm{Eu}^{3+}$ Cubic Centers in the $\mathrm{CaF}_{2}$ Bulk Single Crystal, Phys. Solid State, 2009, 51, 44-49.

[90] V. Möllmann, J. Selling, B. Henke, S. Schweizer, P. Keil, V. Lavin and G. Wortmann, EXAFS Study of the Local Structure at $\mathrm{Eu}^{2+}$ Ions Doped into $\mathrm{CaF}_{2}$, Desy annual report, 2007.

[91] P. Dorenbos, Energy of the First $4 f^{7} \rightarrow 4 f^{6} 5 d$ Transition of $\mathrm{Eu}^{2+}$ in Inorganic Compounds, $J$. Lumin., 2003, 104, 239-260.

[92] G. Blasse, Nature of $\mathrm{Eu}^{2+}$ Luminescence, Phys. Status Solidi B, 1973, 55, K131-K134.

[93] J. D. Axe, 2-Photon Processes in Complex Atoms, Phys. Rev. A, 1964, 136, A42-A45.

[94] N. Bloembergen, The Solved Puzzle of TwoPhoton Rare-Earth Spectra in Solids, J. Lumin., 1984, 31, 23-28.

[95] U. Fritzler and G. Schaack, Investigation of TwoPhoton Absorption in $\mathrm{CaF}_{2}$ and $\mathrm{SrF}_{2}$ Doped with $\mathrm{Eu}^{2+}$, J. Phys. C Solid State Phys., 1976, 9, L23-L26.

[96] U. Fritzler, Investigations on Excited-States of $\mathrm{Eu}^{2+}$ in $\mathrm{CaF}_{2}$-Crystals and $\mathrm{SrF}_{2}$-Crystals by Two-Photon Absorption Spectroscopy, Z. Phys. B, 1977, 27, 289-296.

[97] M. Karbowiak and C. Rudowicz, Trends in Hamiltonian Parameters Determined by Systematic Analysis of f-d Absorption Spectra of Divalent Lanthanides in Alkali-Halides Hosts and
Supported by First Calculations of the $\mathrm{Nd}^{2+}$ Electronic Structure: I. $\mathrm{SrCl}_{2}: \mathrm{Ln}^{2+}$, J. Lumin., 2018, 199, 116-125.

[98] R.-J. Xie, N. Hirosaki and T. Takeda, Wide Color Gamut Backlight for Liquid Crystal Displays Using Three-Band Phosphor-Converted White Light-Emitting Diodes, Appl. Phys. Express, 2009, 2, 022401.

[99] Z. Y. Luo, D. M. Xu and S. T. Wu, Emerging Quantum-Dots-Enhanced LCDs, J. Disp. Technol., 2014, 10, 526-539.

[100] P. Dorenbos, A Review on How Lanthanide Impurity Levels Change with Chemistry and Structure of Inorganic Compounds, ECS J. Solid State Sci. Technol., 2013, 2, R3001-R3011.

[101] B. Ordejón, L. Seijo and Z. Barandiarán, Geometry and Electronic Structure of ImpurityTrapped Excitons in $\mathrm{Cs}_{2} \mathrm{GeF}_{6}: \mathrm{U}^{4+}$ Crystals. The $5 \mathrm{f}^{1} 7 \mathrm{~s}^{1}$ Manifold, J. Chem. Phys., 2007, 126, 194712 .

[102] P. Dorenbos, Relation between $\mathrm{Eu}^{2+}$ and $\mathrm{Ce}^{3+}$ $\mathrm{f} \leftrightarrow \mathrm{d}$-Transition Energies in Inorganic Compounds, J. Phys.: Condens. Matter, 2003, 15, 4797-4807.

[103] J. S. Shi and S. Y. Zhang, Predicting the Position of the Spin-Forbidden Band for $\mathrm{Tb}^{3+}$ Ions in Crystal Hosts, J. Phys.: Condens. Matter, 2003, 15, 4101-4107.

[104] P. Dorenbos, Exchange and Crystal Field Effects on the $4 \mathrm{f}^{n-1} 5 \mathrm{~d}$ Levels of $\mathrm{Tb}^{3+}$, J. Phys.: Condens. Matter, 2003, 15, 6249-6268.

[105] Z. Barandiarán and L. Seijo, Quantum Chemical Analysis of the Bond Lengths in $\mathrm{f}^{n}$ and $\mathrm{f}^{n-1} \mathrm{~d}^{1}$ States of $\mathrm{Ce}^{3+}, \mathrm{Pr}^{3+}, \mathrm{Pa}^{4+}$, and $\mathrm{U}^{4+}$ Defects in Chloride Hosts, J. Chem. Phys., 2003, 119, 3785-3790.

[106] F. Ruiperéz, L. Seijo and Z. Barandiarán, Prediction of Pressure-induced Redshift of $\mathrm{f}^{1} \rightarrow$ $\mathrm{d}(\mathrm{t}(2 \mathrm{~g}))^{1}$ Excitations in $\mathrm{Cs}_{2} \mathrm{NaYCl}_{6}: \mathrm{Ce}^{3+}$ and Its Connection with Bond-length Shortening, $\mathrm{J}$. Chem. Phys., 2005, 122, 234507.

[107] R. Valiente, F. Rodríguez, J. González, H. U. Güdel, R. Martín-Rodríguez, L. Nataf, M. N. Sanz-Ortiz and K. Krämer, High Pressure Optical Spectroscopy of $\mathrm{Ce}^{3+}$-doped $\mathrm{Cs}_{2} \mathrm{NaLuCl}_{6}$, Chem. Phys. Lett., 2009, 481, 149-151.

[108] F. H. Su, W. Chen, K. Ding and G. H. Li, New Observations on the Pressure Dependence of Luminescence from $\mathrm{Eu}^{2+}$-doped $\mathrm{MF}_{2}(\mathrm{M}=\mathrm{Ca}$, Sr, Ba) fluorides, J. Phys. Chem. A, 2008, 112, 4772-4777. 
[109] S. Mahlik, K. Wiśniewski, M. Grinberg and R. S. Meltzer, Temperature and Pressure Dependence of the Luminescence of $\mathrm{Eu}^{2+}$-doped Fluoride Crystals $\mathrm{Ba}_{x} \mathrm{Sr}_{1-x} \mathrm{~F}_{2}(x=0,0.3,0.5$ and 1$)$ : Experiment and Model, J. Phys.: Condens. Matter, 2009, 21, 245601.

[110] C. Chen, K. L. Teo, T. C. Chong, Y. H. Wu, T. S. Low, R. Carey, D. M. Newman, I. Viney, J. P. Wu and J. Bickerton, High-pressure Luminescence Studies in CaS Doped with $\mathrm{Eu}^{2+}, J$. Appl. Phys., 2003, 93, 2559-2561.

[111] P. A. Rodnyi, A. K. Khadro, A. S. Voloshinovski and G. B. Stryganyuk, Europium Luminescence in Fluorite upon High-Energy Excitation, Opt. Spectrosc., 2007, 103, 568-572.

[112] N. Yamashita, Photoluminescence Properties of $\mathrm{Cu}^{+}$Centers in MgS, CaS, SrS and BaS, Jpn. J. Appl. Phys., 1991, 30, 3335-3340.

[113] Python Software Foundation, Python Language Reference, version 2.7., http://www.python. org.

[114] G. Blasse and B. Grabmaier, in Luminescent materials, Springer-Verlag, 1994.

[115] P. A. Tanner, Some Misconceptions Concerning the Electronic Spectra of Tri-Positive Europium and Cerium, Chem. Soc. Rev., 2013, 42, 50905101.

[116] K. Binnemans, Interpretation of Europium(III) Spectra, Coord. Chem. Rev., 2015, 295, 1-45.

[117] C. Cascales, J. Fernández and R. Balda, Investigation of Site-Selective Symmetries of Eu3+ Ions in $\mathrm{KPb}_{2} \mathrm{C}_{l 5}$ by Using Optical Spectroscopy, Opt. Express, 2005, 13, 2141-2152.

[118] C. Cascales, R. Balda, V. Jubera, J. P. Chaminade and J. Fernández, Optical Spectroscopic Study of $\mathrm{Eu}^{3+}$ Crystal Field Sites in $\mathrm{Na}_{3} \mathrm{La}_{9} \mathrm{O}_{3}\left(\mathrm{BO}_{3}\right)_{8}$ Crystal, Opt. Express, 2008, 16, 2653-2662.

[119] V. A. Morozov, A. Bertha, K. W. Meert, S. Van Rompaey, D. Batuk, G. T. Martinez, S. Van Aert, P. F. Smet, M. V. Raskina, D. Poelman, A. M. Abakumov and J. Hadermann, Incommensurate Modulation and Luminescence in the $\mathrm{CaGd}_{2(1-x)} \mathrm{Eu}_{2 x}\left(\mathrm{MoO}_{4}\right)_{4(1-y)}\left(\mathrm{WO}_{4}\right)_{4 y}(0 \leq$ $x \leq 1,0 \leq y \leq 1)$ Red Phosphors, Chem. Mater., 2013, 25, 4387-4395.

[120] J. Sierro, Paramagnetic Resonance of $\mathrm{Gd}^{3+}$ in $\mathrm{SrF}_{2}$ and $\mathrm{BaF}_{2}$, Phys. Lett., 1963, 4, 178-180.

[121] B. P. Zakharchenya and I. B. Rusanov, Experimental Proof of Existence of Strictly Cubic Centers in a $\mathrm{CaF}_{2}: \mathrm{Eu}^{3+}$ Crystal, Sov. Phys. - Solid State, $1966, \mathbf{8}, 31$.
[122] E. Friedman and W. Low, Effect of Thermal Treatment of Paramagnetic Resonance Spectra of Rare Earth Impurities in Calcium Fluoride, $J$. Chem. Phys., 1960, 33, 1275-1276.

[123] R. J. Hamers, J. R. Wietfeldt and J. C. Wright, Defect Chemistry in $\mathrm{CaF}_{2}: \mathrm{Eu}^{3+}$, J. Chem. Phys., 1982, 77, 683-692.

[124] R. Pandey and S. Sivaraman, Spectroscopic Properties of Defects in Alkaline-Earth Sulfides, J. Phys. Chem. Solids, 1991, 52, 211-225.

[125] J. Kreissl, Electron-Paramagnetic-Resonance of $\mathrm{Ce}^{3+}$ in SrS Powder, Phys. Status Solidi B, 1993, 180, 441-443.

[126] M. Albin and W. D. Horrocks, Europium(III) Luminescence Excitation Spectroscopy. Quantitive Correlation between the Total Charge on the Ligands and the ${ }^{7} \mathrm{~F}_{0} \rightarrow{ }^{5} \mathrm{D}_{0}$ Transition Frequency in Europium(III) Complexes, Inorg. Chem., 1985, 24, 895-900.

[127] S. T. Frey and W. D. Horrocks, On Correlating the Frequency of the ${ }^{7} \mathrm{~F}_{0} \rightarrow{ }^{5} \mathrm{D}_{0}$ Transition in $\mathrm{Eu}^{3+}$ Complexes with the Sum of 'Nephelauxetic Parameters' for all of the Coordinating Atoms, Inorg. Chim. Acta, 1995, 229, 383 - 390.

[128] G. R. Choppin and Z. M. Wang, Correlation between Ligand Coordination Number and the Shift of the ${ }^{7} \mathrm{~F}_{0^{-}}{ }^{5} \mathrm{D}_{0}$ Transition Frequency in Europium(III) Complexes, Inorg. Chem., 1997, 36, 249-252.

[129] P. A. Tanner, Y. Y. Yeung and L. X. Ning, What Factors Affect the ${ }^{5} \mathrm{D}_{0}$ Energy of $\mathrm{Eu}^{3+}$ ? An Investigation of Nephelauxetic Effect, J. Phys. Chem. A, 2013, 117, 2771-2781.

[130] J. J. Joos, L. Seijo and Z. Barandiarán, Direct Evidence of Intervalence Charge-Transfer States of Eu-Doped Luminescent Materials, J. Phys. Chem. Lett., 2019, 10, 1581-1586.

[131] A. Jain, S. P. Ong, G. Hautier, W. Chen, W. D. Richards, S. Dacek, S. Cholia, D. Gunter, D. Skinner, G. Ceder and K. A. Persson, The Materials Project: A Materials Genome Approach to Accelerating Materials Innovation, APL Mater., 2013, 1, 011002.

[132] M. de Jong, W. Chen, T. Angsten, A. Jain, R. Notestine, A. Gamst, M. Sluiter, C. A. Krishna, S. van der Zwaag, J. J. Plata, C. Toher, S. Curtarolo, G. Ceder, K. A. Persson and M. Asta, Charting the Complete Elastic Properties of Inorganic Crystalline Compounds, Sci. Data, 2015, 2, 150009. 